\title{
On the structure of inhomogeneous quantum groups
}

\author{
P. Podleś ${ }^{1 * \dagger}$ and S. L. Woronowicz ${ }^{2 \ddagger}$ \\ ${ }^{1}$ Department of Mathematics, University of California \\ Berkeley CA 94720, USA \\ ${ }^{2}$ Department of Mathematical Methods in Physics \\ Faculty of Physics, University of Warsaw \\ Hoża 74, 00-682 Warszawa, Poland
}

\begin{abstract}
We investigate inhomogeneous quantum groups $G$ built from a quantum group $H$ and translations. The corresponding commutation relations contain inhomogeneous terms. Under certain conditions (which are satisfied in our study of quantum Poincaré groups [12]) we prove that our construction has correct 'size', find the $R$-matrices and the analogues of Minkowski space for $G$.
\end{abstract}

\section{Introduction}

Inhomogeneous quantum groups, their homogeneous spaces and corresponding R-matrices were studied by many authors (cf e.g. [2], [7], [4], [13], [9], [8], [3]). Here we propose a general construction which covers the examples [7],

*On leave from Department of Mathematical Methods in Physics, Faculty of Physics, University of Warsaw, Hoża 74, 00-682 Warszawa, Poland

${ }^{\dagger}$ This research was supported in part by NSF grant DMS92-43893 and in part by Polish KBN grant No 2 P301 02007

†This research was supported by Polish KBN grant No 2 P301 02007 
[3] and is suitable for our study of quantum Poincaré groups (without dilatations) [12]. We work in the framework of Hopf algebras treated as algebras of functions on quantum groups. In Section 1 we assume that $G$ is an inhomogeneous quantum group built from a quantum group $H$ and translations described by the elements $p_{i}$ corresponding to an irreducible representation $\Lambda$ of $H$. The commutation relations can contain inhomogeneous terms. It turns out that the leading terms in these relations are governed by the structure of certain bicovariant bimodule of $H$. In particular, the leading terms in relations among $p_{i}$ must correspond to the eigenvalue -1 of the corresponding R-matrix $R$ (cf [11], [8]). In Section 2 we add the condition that all the representations of $H$ are completely reducible (which is the case in [12] when $H$ is a quantum Lorentz group [16]) and find the commutation relations between functions on $H$ and $p_{i}$. In Section 3 we assume that $R^{2}=\mathbf{1}$ (or $(R-Q \mathbf{1})(R+\mathbf{1})=0$ where $Q \neq 0$ is not a root of unity) and that we have as many quadratic relations among $p_{i}$ as it is allowed by the eigenvalue -1 property (so, if there would be no inhomogeneous terms, $p_{i}$ would be $R$-symmetric). That is the simplest case which is sufficient in [12]. We find the exact form of commutation relations and the necessary and sufficient conditions for the corresponding coefficients. If they are fulfilled, there are no relations of higher order and our construction has the same 'size' as in the absence of inhomogeneous terms. The R-matrices for the fundamental representation of $G$ are classified. In Section 4 we consider the $*$-structure and isomorphisms among our objects. In Section 5 we prove that (under some conditions which are fulfilled in [12]) each $G$ possesses exactly one analogue of Minkowski space. Inhomogeneous Poisson groups are considered in [18].

For the simplicity of calculations, the small Latin indices $a, b, c, d, \ldots$, belong to a finite set $\mathcal{I}$ in Sections $1-5$. We sum over repeated indices which are not taken in brackets (Einstein's convention). The number of elements in a set $B$ is $\# B$ or $|B|$. We work over the field $\mathbf{C}$. Unit matrix with dimension $N$ is denoted by $\mathbf{1}_{N}$. If $V, W$ are vector spaces then $\tau_{V W}: V \otimes W \longrightarrow W \otimes V$ is given by $\tau_{V W}(x \otimes y)=y \otimes x, x \in V, y \in W$. We often write $\tau$ instead of $\tau_{V W}$. If $\mathcal{A}$ is a linear space and $v, v^{\prime} \in M_{N}(\mathcal{A}), N \in \mathbf{N}$, then $v \oplus v^{\prime} \in M_{N}(\mathcal{A} \otimes \mathcal{A})$ is defined by $\left(v \oplus v^{\prime}\right)_{i j}=v_{i k} \otimes v^{\prime}{ }_{k j}, i, j=1, \ldots, N$ (Einstein's convention!). If moreover $\mathcal{A}$ is an algebra, $v \in M_{N}(\mathcal{A}), w \in M_{K}(\mathcal{A})$, then $v \oplus w \in M_{N K}(\mathcal{A})$ is defined by $(v \bigcirc w)_{i j, k l}=v_{i k} w_{j l}, i, k=1, \ldots, N, j, l=1, \ldots, K$. We use the abbreviation $v^{\oplus n}$ for $v \oplus \ldots$. D $v$ ( $n$ times). If $\mathcal{A}=\mathbf{C}$ we may write $\otimes$ 
instead of $\mathbb{1}$. If also $v^{\prime} \in M_{N}(\mathcal{A}), w^{\prime} \in M_{K}(\mathcal{A})$ then $(v \oplus w) \oplus\left(v^{\prime} \oplus w^{\prime}\right)=$ $\left(v \oplus v^{\prime}\right) \oplus\left(w \oplus w^{\prime}\right)$ (see $(2.18)$ of [14]). If $\mathcal{A}$ is a ${ }^{*}$-algebra then the conjugate of $v$ is defined as $\bar{v} \in M_{N}(\mathcal{A})$, where $\bar{v}_{i j}=v_{i j}{ }^{*}, i, j=1, \ldots, N$.

Throughout the paper quantum groups $G$ are abstract objects described by the corresponding Hopf $\left({ }^{*}-\right)$ algebras $\operatorname{Poly}(G)=(\mathcal{A}, \Delta)$. We denote by $\Delta, \epsilon, S$ the comultiplication, counit and coinverse of $\operatorname{Poly}(G)$. We always assume that $S$ is invertible. We say that $v$ is a representation of $G$ (i.e. $v \in \operatorname{Rep} G)$ if $v \in M_{N}(\mathcal{A}), N \in \mathbf{N}$, and $\Delta v_{i j}=v_{i k} \otimes v_{k j}, \epsilon\left(v_{i j}\right)=\delta_{i j}$, $i, j=1,2, \ldots, N$. Then $\operatorname{dim} v=N$. The conjugate of a representation and tensor product of representations are also representations. Matrix elements of representations of $G$ span $\mathcal{A}$ as a linear space. The set of nonequivalent irreducible representations of $G$ is denoted by $\operatorname{Irr} G$. If $v, w \in \operatorname{Rep} G$ then we say that $A \in M_{\operatorname{dim} w \times \operatorname{dim} v}(\mathbf{C})$ intertwines $v$ with $w$ (i.e. $A \in \operatorname{Mor}(v, w)$ ) if $A v=w A$. We use the following notations:

$$
a * \rho=(\rho \otimes \mathrm{id}) \Delta a, \quad \rho * a=(\mathrm{id} \otimes \rho) \Delta a, \quad \rho * \rho^{\prime}=\left(\rho \otimes \rho^{\prime}\right) \Delta
$$

for $a \in \mathcal{A}, \rho, \rho^{\prime} \in \mathcal{A}^{\prime}$. Let us recall the following well-known

Proposition 0.1 Let $\mathcal{A}$ be an augmented algebra (i.e. a unital algebra endowed with a unital homomorphism $\epsilon: \mathcal{A} \longrightarrow \mathbf{C}$ ) and $\omega_{i}, i=1,2, \ldots, M$, and $\eta_{j}, j=1,2, \ldots, N$, be two free bases of the left $\mathcal{A}$-module $\Gamma$. Then $M=N$.

Proof. One has $\omega_{i}=a_{i j} \eta_{j}, \eta_{j}=b_{j i} \omega_{i}$ for some $a_{i j}, b_{j i} \in \mathcal{A}$. It follows $a_{i j} b_{j k}=\delta_{i k} I, b_{l i} a_{i j}=\delta_{l j} I, k=1, \ldots, M, l=1, \ldots, N$. Applying $\epsilon$, one gets $\epsilon(a) \epsilon(b)=\mathbf{1}_{M}, \epsilon(b) \epsilon(a)=\mathbf{1}_{N}$, hence $\epsilon(a)$ is invertible, $M=N$.

Therefore the dimension $\operatorname{dim}_{\mathcal{A}}$ of a free left $\mathcal{A}$-module (where $\mathcal{A}$ is a Hopf algebra) is well defined. We can use the above notions and facts (if applicable) also for general $\left({ }^{*}-\right.$ ) bialgebras (without $S$ ) and not necessarily quadratic matrices.

\section{Inhomogeneous quantum groups}

In this Section we define inhomogeneous quantum groups and study leading terms in their commutation relations using the theory of bicovariant bimodules [15]. The importance of left covariant bimodule structure in investigation of inhomogeneous quantum groups was first noticed in [13]. 
Let us assume that $\operatorname{Poly}(H)=(\mathcal{A}, \Delta)$ is a Hopf algebra with a distinguished irreducible representation $\Lambda=\left(\Lambda_{r s}\right)_{r, s \in \mathcal{I}}$ of $H,|\mathcal{I}|<\infty$. We set $\mathbf{1}=\mathbf{1}_{|\mathcal{I}|}$. We shall consider bialgebras $\operatorname{Poly}(G)=(\mathcal{B}, \Delta)$ such that:

1. $\mathcal{B}$ is generated (as algebra) by $\mathcal{A}$ and the elements $p_{s}, s \in \mathcal{I}$.

2. $\mathcal{A}$ is a sub-bialgebra of $\mathcal{B}$.

3. $\mathcal{P}=\left(\begin{array}{cc}\Lambda & p \\ 0 & I\end{array}\right)$ is a representation of $G$.

4. There exists $i \in \mathcal{I}$ such that $p_{i} \notin \mathcal{A}$.

5. $\Gamma \mathcal{A} \subset \Gamma$ where $\Gamma=\mathcal{A} \cdot X+\mathcal{A}, X=\operatorname{span}\left\{p_{i}, \quad i \in \mathcal{I}\right\}$.

Due to 5 ., $\Gamma$ is an $\mathcal{A}$-bimodule. In virtue of 2.-3., $\Delta \mathcal{A} \subset \mathcal{A} \otimes \mathcal{A}$,

$$
\Delta p=\Lambda \oplus p+p \oplus I,
$$

hence $\Delta \Gamma \subset \mathcal{A} \otimes \Gamma+\Gamma \otimes \mathcal{A}$. We define bimodule $\dot{\Gamma}=\Gamma / \mathcal{A}$ by $a \dot{\omega}=a \dot{\omega}$, $\dot{\omega} a=\dot{\omega} a$ where $\dot{\omega}$ is the element of $\dot{\Gamma}$ corresponding to $\omega \in \Gamma, a \in \mathcal{A}$. We see that $\Delta$ induces a linear mapping

$$
\dot{\Delta}: \dot{\Gamma} \longrightarrow(\mathcal{A} \otimes \Gamma+\Gamma \otimes \mathcal{A}) /(\mathcal{A} \otimes \mathcal{A}) \approx(\mathcal{A} \otimes \dot{\Gamma}) \oplus(\dot{\Gamma} \otimes \mathcal{A}) .
$$

We get the decomposition $\dot{\Delta}=\Delta_{L}+\Delta_{R}, \Delta_{L}: \dot{\Gamma} \longrightarrow \mathcal{A} \otimes \dot{\Gamma}, \Delta_{R}: \dot{\Gamma} \longrightarrow \dot{\Gamma} \otimes \mathcal{A}$. In particular, $\Delta_{L} \dot{p_{s}}=\Lambda_{s t} \otimes \dot{p_{t}}, \Delta_{R} \dot{p_{s}}=\dot{p_{s}} \otimes I$. Using the properties of $\Delta$, one can easily check that $\left(\dot{\Gamma}, \Delta_{L}, \Delta_{R}\right)$ is a bicovariant bimodule (cf $[15]$, Definition 2.3 and a similar argument in the proof of Theorem 5 of $[1])$. We notice that $\dot{p}_{s}(s \in \mathcal{I})$ are elements in the set $\dot{\Gamma}_{i n v}$ of right-invariant elements of $\dot{\Gamma}$. Moreover, under $\Delta_{L}$ they transform according to an irreducible representation $\Lambda$ and at least one of them is nonzero (the condition 4.). Thus they are linearly independent. They generate (see the condition 5.) the left module $\dot{\Gamma}$. Using Theorem 2.3 of [15], we get that $\dot{p}_{s}(s \in \mathcal{I})$ form a linear basis of $\dot{\Gamma}_{i n v}$ and thus a basis of the left module $\dot{\Gamma}$. Moreover, the same Theorem implies that

$$
\dot{p_{s}} a=\left(a * f_{s t}\right) \dot{p_{t}}
$$

for some functionals $f_{s t} \in \mathcal{A}^{\prime}$ such that

$$
f_{s t}(a b)=f_{s m}(a) f_{m t}(b), \quad a, b \in \mathcal{A}, \quad f_{s t}(I)=\delta_{s t} .
$$

\footnotetext{
${ }^{1}$ there is a missprint in (2.33) of [15], to get the correct form one has to replace $f_{i j}$ by $f_{i j} \circ \kappa^{-2}$, which is denoted by $f_{i j}$ in the present paper (cf [1]])
} 
(It implies

$$
f_{a b} \circ S * f_{b c}=f_{a b} * f_{b c} \circ S=\delta_{a c} \epsilon
$$

- one can apply both sides to $v_{i j}, v \in \operatorname{Rep} \mathcal{A}$, which span $\mathcal{A}$ ). Applying $\Delta_{L}$ to $(1.2)$, we get

$$
\begin{gathered}
\Lambda_{s t} a^{(1)} \otimes\left(a^{(2)} * f_{t r}\right) \dot{p_{r}}=\left(\Lambda_{s t} \otimes \dot{p}_{t}\right) \Delta a= \\
\Delta\left(a * f_{s t}\right)\left(\Lambda_{t r} \otimes \dot{p_{r}}\right)=\left(a^{(1)} * f_{s t}\right) \Lambda_{t r} \otimes a^{(2)} \dot{p}_{r},
\end{gathered}
$$

where we denoted $\Delta a=a^{(1)} \otimes a^{(2)}$. Comparing the coefficients multiplying $\dot{p_{r}}$ and applying id $\otimes \epsilon$, one obtains

$$
\Lambda_{s t}\left(f_{t r} * a\right)=\left(a * f_{s t}\right) \Lambda_{t r}, \quad a \in \mathcal{A}
$$

Let us pass to $\Gamma$. The elements $\left\{I, p_{s}: \quad s \in \mathcal{I}\right\}$ form a basis of $\Gamma$ as a left module. Moreover, (1.2) implies

$$
p_{s} a=\left(a * f_{s t}\right) p_{t}+\phi_{s}(a), \quad a \in \mathcal{A},
$$

for some $\phi_{s}: \mathcal{A} \longrightarrow \mathcal{A}$. Using $p_{s}(a b)=\left(p_{s} a\right) b, p_{s} I=p_{s}$ and (1.3), one gets

$$
\phi_{s}(a b)=\left(a * f_{s t}\right) \phi_{t}(b)+\phi_{s}(a) b, \quad a, b \in \mathcal{A}, \quad \phi_{s}(I)=0 .
$$

Thus the mapping

$$
\psi: \mathcal{A} \ni a \longrightarrow\left(\begin{array}{cc}
a * f & \phi(a) \\
0 & a
\end{array}\right) \in M_{|\mathcal{I}|+1}(\mathcal{A})
$$

is a unital homomorphism. Applying $\Delta$ to (1.6), one gets the equality of both sides if and only if (1.5) and

$$
\Delta \phi_{s}(a)=\left(\Lambda_{s t} \otimes I\right)\left[\left(\mathrm{id} \otimes \phi_{t}\right) \Delta(a)\right]+\left(\phi_{s} \otimes \mathrm{id}\right) \Delta a, \quad a \in \mathcal{A},
$$

hold.

Before investigation (for certain class of $\mathcal{A}$ ) of this equation let us consider the general situation. We assume that $f_{s t} \in \mathcal{A}^{\prime}$ and $\phi_{s}: \mathcal{A} \longrightarrow \mathcal{A}$ satisfy (1.3), (1.5), (1.7) and (1.9). Let $\tilde{\mathcal{B}}$ be the algebra with $I$ generated by $\mathcal{A}$ and $\tilde{p}_{s}, s \in \mathcal{I}$, with relations (1.6). We set $\tilde{p}_{K}=\tilde{p}_{k_{1}} \cdot \ldots \cdot \tilde{p}_{k_{n}}$ for $K=$ $\left(k_{1}, \ldots, k_{n}\right) \in \mathcal{I}_{n}=\mathcal{I} \times \ldots \times \mathcal{I}(n$ times $), \hat{\mathcal{I}}=\sqcup_{n=0}^{\infty} \mathcal{I}_{n}\left(\tilde{p}_{\emptyset}=I\right)$. 
Lemma $1.1 \tilde{p}_{K}, K \in \hat{\mathcal{I}}$, form a basis of the left $\mathcal{A}$-module $\tilde{\mathcal{B}}$.

Proof. We define $\mathcal{C}$ as a free left $\mathcal{A}$-module with basis $P_{K}=p_{k_{1}} \otimes \ldots \otimes p_{k_{n}}$ for $K=\left(k_{1}, \ldots, k_{n}\right) \in \hat{\mathcal{I}}$. We also set $\mathcal{C}_{n}=\mathcal{A} \cdot \operatorname{span}\left\{P_{K}: K \in \mathcal{I}_{n}\right\}$ and introduce linear mappings $\lambda_{n}: \mathcal{C}_{n} \otimes \mathcal{A} \longrightarrow \mathcal{C}_{n}$ by $\lambda_{0}\left(b P_{\emptyset} \otimes a\right)=b a P_{\emptyset}$,

$$
\lambda_{n}\left(b\left(P_{K} \otimes p_{i}\right) \otimes a\right)=\lambda_{n-1}\left(b P_{K} \otimes\left(a * f_{i s}\right)\right) \otimes p_{s}+\lambda_{n-1}\left(b P_{K} \otimes \phi_{i}(a)\right),
$$

$K \in \mathcal{I}_{n-1}$. Next we define linear mapping (multiplication) $m: \mathcal{C} \otimes \mathcal{C} \longrightarrow \mathcal{C}$ in $\mathcal{C}$ by $m\left(b P_{K} \otimes a P_{L}\right)=\lambda_{n}\left(b P_{K} \otimes a\right) \otimes P_{L}$ for $K, L \in \hat{\mathcal{I}}$. After some calculations (using (1.7)) one can check that $(\mathcal{C}, m)$ is an algebra with identity $P_{\emptyset}$. Moreover, there exists a unital homomorphism $\rho: \tilde{\mathcal{B}} \longrightarrow \mathcal{C}$ given by $\rho\left(a \tilde{p}_{K}\right)=a P_{K}((1.6)$ holds in $\mathcal{C})$. But $P_{K}$ form a basis of $\mathcal{C}$ and hence $\tilde{p}_{K}$ are independent (over $\mathcal{A}$ ) in $\tilde{\mathcal{B}}$. They also generate (due to $(1.6)) \tilde{\mathcal{B}}$ as the left module.

Let the comultiplication in $\tilde{\mathcal{B}}$ be given by the comultiplication in $\mathcal{A}$ and (1.1) (it is well defined due to (1.5) and (1.9) - see remarks before (1.9)). Then $\tilde{\mathcal{B}}$ is a bialgebra with a natural bialgebra epimorphism $\pi: \tilde{\mathcal{B}} \longrightarrow \mathcal{B}$ given by $\pi(a)=a, a \in \mathcal{A}, \pi\left(\tilde{p}_{s}\right)=p_{s}$.

We set $J=\operatorname{ker} \pi, \tilde{\Gamma}=\mathcal{A} \cdot \operatorname{span}\left\{\tilde{p}_{s}, \quad s \in \mathcal{I}\right\}, \tilde{\mathcal{B}}_{k}=\mathcal{A} \cdot \operatorname{span}\left\{\tilde{p}_{J}, \quad J \in \mathcal{I}_{k}\right\}$ (with basis $\tilde{p}_{J}, J \in \mathcal{I}_{k}$ ), $\tilde{\mathcal{B}}^{k}=\oplus_{l=0}^{k} \tilde{\mathcal{B}}_{l}$ (cf. Lemma 1.1), $J^{k}=\tilde{\mathcal{B}}^{k} \cap J$. Let $J_{k}$ be some vector space such that $J^{k-1} \oplus J_{k}=J^{k}, k \in \mathbf{N}\left(J_{0}=\{0\}\right)$. We have $J_{k} \cap \tilde{\mathcal{B}}^{k-1} \subset J_{k} \cap J^{k-1}=\{0\}$, so we can define $\tilde{\mathcal{B}}_{(k)}$ as some vector space such that $\tilde{\mathcal{B}}^{k-1} \oplus J_{k} \oplus \tilde{\mathcal{B}}_{(k)}=\tilde{\mathcal{B}}^{k}$. In particular, we can put $J^{0}=J^{1}=J_{0}=J_{1}=$ $\{0\}, J_{2}=J^{2}, \tilde{\mathcal{B}}_{(0)}=\tilde{\mathcal{B}}_{0}=\mathcal{A}, \tilde{\mathcal{B}}_{(1)}=\tilde{\mathcal{B}}_{1}=\tilde{\Gamma}$.

We shall investigate $J_{2}$. Let $s \in J_{2}$. Then $s \in J,(\pi \otimes \pi) \Delta s=\Delta \pi s=0$, $\Delta s \in \tilde{\mathcal{B}} \otimes J+J \otimes \tilde{\mathcal{B}}$. But also $s \in \tilde{\mathcal{B}}^{2}, \Delta s \in \tilde{\mathcal{B}}^{2} \otimes \mathcal{A}+\mathcal{A} \otimes \tilde{\mathcal{B}}^{2}+\tilde{\mathcal{B}}^{1} \otimes \tilde{\mathcal{B}}^{1}$. Using $\tilde{\mathcal{B}}=\left(\oplus J_{k}\right) \oplus\left(\oplus \tilde{\mathcal{B}}_{(k)}\right), J=\oplus J_{k}, \tilde{\mathcal{B}}^{2}=\tilde{\mathcal{B}}_{(0)} \oplus \tilde{\mathcal{B}}_{(1)} \oplus \tilde{\mathcal{B}}_{(2)} \oplus J_{2}, \tilde{\mathcal{B}}^{1}=\tilde{\mathcal{B}}_{(0)} \oplus \tilde{\mathcal{B}}_{(1)}$, one gets

$$
\Delta s \in \mathcal{A} \otimes J_{2} \oplus J_{2} \otimes \mathcal{A} .
$$

We put $\dot{\Gamma}_{2}=\tilde{\mathcal{B}}^{2} / \tilde{\mathcal{B}}^{1}$. We see that $\Delta$ induces a linear mapping

$$
\begin{gathered}
\Delta_{2}: \dot{\Gamma}_{2} \longrightarrow\left(\tilde{\mathcal{B}}^{2} \otimes \mathcal{A}+\mathcal{A} \otimes \tilde{\mathcal{B}}^{2}+\tilde{\mathcal{B}}^{1} \otimes \tilde{\mathcal{B}}^{1}\right) /\left(\tilde{\mathcal{B}}^{1} \otimes \mathcal{A}+\mathcal{A} \otimes \tilde{\mathcal{B}}^{1}\right) \approx \\
\dot{\Gamma}_{2} \otimes \mathcal{A} \oplus \mathcal{A} \otimes \dot{\Gamma}_{2} \oplus \dot{\Gamma} \otimes \dot{\Gamma} .
\end{gathered}
$$

We get the decomposition $\Delta_{2}=\Delta_{2 L}+\Delta_{2 R}+\tilde{\Delta}, \Delta_{2 L}: \dot{\Gamma}_{2} \longrightarrow \mathcal{A} \otimes \dot{\Gamma}_{2}$, $\Delta_{2 R}: \dot{\Gamma}_{2} \longrightarrow \dot{\Gamma}_{2} \otimes \mathcal{A}$ and $\tilde{\Delta}: \dot{\Gamma}_{2} \longrightarrow \dot{\Gamma} \otimes \dot{\Gamma}$. 
Lemma 1.2 The elements $\left[\tilde{p}_{i} \tilde{p}_{j}\right]$ form a linear basis of $\left(\dot{\Gamma}_{2}\right)_{\text {inv }}$, hence a basis of the left module $\dot{\Gamma}_{2}$, while $\dot{p}_{i} \otimes \dot{p}_{j}$ form a linear basis of $\left(\dot{\Gamma} \otimes_{\mathcal{A}} \dot{\Gamma}\right)_{\text {inv }}$, hence a basis of the left module $\dot{\Gamma} \otimes_{\mathcal{A}} \dot{\Gamma}$.

Moreover, $\xi: \dot{\Gamma}_{2} \longrightarrow \dot{\Gamma} \otimes_{\mathcal{A}} \dot{\Gamma}$ given by $\xi\left(\left[\tilde{p}_{i} \tilde{p}_{j}\right]\right)=\dot{p}_{i} \otimes \dot{p}_{j}$ defines an isomorphism of bicovariant bimodules.

Proof. The elements $\left[\tilde{p}_{i} \tilde{p}_{j}\right]$ are basis of the left module $\dot{\Gamma}_{2}=\tilde{\mathcal{B}}^{2} / \tilde{\mathcal{B}}^{1}$ (Lemma 1.1) and belong to $\left(\dot{\Gamma}_{2}\right)_{i n v}$ while $\dot{p}_{i} \otimes \dot{p}_{j}$ are basis of the left module $\dot{\Gamma} \otimes_{\mathcal{A}} \dot{\Gamma}$ (they are linearly independent elements of $\left(\dot{\Gamma} \otimes_{\mathcal{A}} \dot{\Gamma}\right)_{i n v}$ and generate the left module). Moreover,

$$
\begin{gathered}
{\left[\tilde{p}_{i} \tilde{p}_{j}\right] a=\left(a * f_{j s} * f_{i m}\right)\left[\tilde{p}_{m} \tilde{p}_{s}\right],} \\
\Delta_{2 L}\left[\tilde{p}_{i} \tilde{p}_{j}\right]=\Lambda_{i m} \Lambda_{j s} \otimes\left[\tilde{p}_{m} \tilde{p}_{s}\right], \\
\Delta_{2 R}\left[\tilde{p}_{i} \tilde{p}_{j}\right]=\left[\tilde{p}_{i} \tilde{p}_{j}\right] \otimes I,
\end{gathered}
$$

and similarly for $\dot{p_{i}} \otimes \dot{p_{j}}$. Thus $\left(\dot{\Gamma}_{2}, \Delta_{2 L}, \Delta_{2 R}\right)$ is a bicovariant bimodule isomorphic (by $\xi$ ) to $\dot{\Gamma} \otimes_{\mathcal{A}} \dot{\Gamma}$.

In the following we shall identify $x$ with $\xi(x)$ and $\dot{\Gamma}_{2}$ with $\dot{\Gamma} \otimes_{\mathcal{A}} \dot{\Gamma}$. Let us recall that there exists a unique bicovariant bimodule isomorphism $\rho$ : $\dot{\Gamma} \otimes_{\mathcal{A}} \dot{\Gamma} \longrightarrow \dot{\Gamma} \otimes_{\mathcal{A}} \dot{\Gamma}$ given by $\rho(\eta \otimes \omega)=\omega \otimes \eta$ where $\eta$ is a right-invariant, while $\omega$ is a left-invariant element of $\dot{\Gamma}\left(\rho=\sigma^{-1}\right.$ where $\sigma$ is given in Proposition 3.1 of [15]). Thus $\operatorname{ker}(\rho+\mathrm{id})$ is a bicovariant subbimodule of $\dot{\Gamma} \otimes_{\mathcal{A}} \dot{\Gamma}$.

We define

$$
R_{i j, s m}=f_{i m}\left(\Lambda_{j s}\right)
$$

Setting $a=\Lambda_{m n}$ in (1.5), we get

$$
R \in \operatorname{Mor}(\Lambda \oplus \Lambda, \Lambda \oplus \Lambda) .
$$

Due to

$$
\rho\left(\dot{p}_{i} \otimes \dot{p}_{j}\right)=f_{i m}\left(\Lambda_{j s}\right) \dot{p_{s}} \otimes p_{m}
$$

(similar proof as for (3.15) of [15]) $R^{T}$ is the matrix of $\rho$ for the basis $\dot{p}_{i} \otimes \dot{p}_{j}$ $(i, j \in \mathcal{I})$ of $\left(\dot{\Gamma}_{2}\right)_{i n v}$.

Proposition 1.3 Let $K$ be a right-covariant left submodule of $\dot{\Gamma}_{2}, N=$ $\operatorname{dim} K \in \mathbf{N}$, and

$$
a_{i j}^{\alpha}\left(\dot{p}_{i} \otimes \dot{p}_{j}\right), \quad \alpha=1, \ldots, N, \text { form a linear basis of } K_{i n v}
$$


$\left(a_{i j}^{\alpha} \in \mathbf{C}\right)$. Then $K$ is a bicovariant bimodule iff there exists $g=\left(g_{\alpha \beta}\right)_{\alpha, \beta=1}^{N} \in$ Rep $H$ such that

$$
a(\Lambda \oplus \Lambda)=g \cdot a
$$

(we set $a_{\alpha, m n}=a_{m n}^{\alpha}$ ) and $\omega_{\alpha \beta} \in \mathcal{A}^{\prime}, \alpha, \beta=1, \ldots, N$, such that

$$
\begin{gathered}
a_{i j}^{\alpha}\left(f_{j s} * f_{i m}\right)=\omega_{\alpha \beta} a_{m s}^{\beta}, \\
\omega_{\alpha \gamma}(b c)=\omega_{\alpha \beta}(b) \omega_{\beta \gamma}(c) \quad(b, c \in \mathcal{A}), \quad \omega_{\alpha \beta}(I)=\delta_{\alpha \beta} .
\end{gathered}
$$

In that case $g$ is a quotient representation of $\Lambda \oplus \Lambda$, corresponding to $K_{i n v}$ :

$$
\Delta_{2 L}\left[a_{i j}^{\alpha} \tilde{p}_{i} \tilde{p}_{j}\right]=g_{\alpha \beta} \otimes\left[a_{m n}^{\beta} \tilde{p}_{m} \tilde{p}_{n}\right] .
$$

Moreover, $K \subset \operatorname{ker}(\rho+\mathrm{id})$ iff

$$
a^{\alpha}\left(R+\mathbf{1}^{\otimes 2}\right)=0,
$$

where $\left(a^{\alpha}\right)_{m n}=a_{m n}^{\alpha}$.

Proof. If $K$ is a bicovariant bimodule then $\Delta_{2 L} K_{\text {inv }} \subset \mathcal{A} \otimes K_{\text {inv }}$. Therefore there exist $g_{\alpha \beta} \in \mathcal{A}$ such that (1.18) holds. Using the definition and properties of $\Delta_{2 L}$, one gets (1.15) and that $g$ is a representation of $H$. Conversely, (1.15) gives (1.18) and left invariance of $K$. Moreover, the right module condition for $K$ means that for any $b \in \mathcal{A}$

$$
\begin{aligned}
a_{i j}^{\alpha}\left[\tilde{p}_{i} \tilde{p}_{j}\right] b= & a_{i j}^{\alpha}\left(b * f_{j s} * f_{i m}\right)\left[\tilde{p}_{m} \tilde{p}_{s}\right]= \\
& b_{\alpha \beta} a_{m s}^{\beta}\left[\tilde{p}_{m} \tilde{p}_{s}\right]
\end{aligned}
$$

for some $b_{\alpha \beta} \in \mathcal{A}$. Setting $\omega_{\alpha \beta}(b)=e\left(b_{\alpha \beta}\right)$ we get $\omega_{\alpha \beta} \in \mathcal{A}^{\prime}$ and (1.16)-(1.17) (we use $(b c)_{\alpha \gamma}=b_{\alpha \beta} c_{\beta \gamma}, I_{\alpha \beta}=\delta_{\alpha \beta} I$ ). Conversely, (1.16)-(1.17) give the right module condition for $K$.

Due to (1.15) $\mathrm{g}$ is a quotient representation (see Appendix B of [6]) of $\Lambda \oplus \Lambda$ (due to (1.14), $a$ is surjective).

Finally, (1.13) and (11.11) give

$$
(\rho+\mathrm{id})\left(a_{i j}^{\alpha} \dot{p}_{i} \otimes \dot{p}_{j}\right)=a_{i j}^{\alpha}\left(R_{i j, s m}+\delta_{i s} \delta_{j m}\right) \dot{p}_{s} \otimes \dot{p}_{m}
$$

and the last statement holds.

From now on we set $K=J_{2} / \tilde{\mathcal{B}}^{1} \subset \dot{\Gamma}_{2}$. As left modules $K \approx J_{2}$ since $J_{2} \cap \tilde{\mathcal{B}}^{1}=\{0\}$. We shall see that $K$ satisfies all the conditions of Proposition 1.3: 
Proposition $1.4 K$ is a bicovariant subbimodule of $\operatorname{ker} \tilde{\Delta}=\operatorname{ker}(\rho+\mathrm{id}) \subset$ $\dot{\Gamma} \otimes_{\mathcal{A}} \dot{\Gamma}=\dot{\Gamma}_{2}$.

Remark 1.5 Thus in interesting situations $\rho$ should have an eigenvalue -1 (cf [1], [8]).

Proof. Since $J$ is an ideal, $J_{2}$ is a bimodule, so is $K$. Due to (1.10), $\Delta_{2 L} K \subset$ $\mathcal{A} \otimes K, \Delta_{2 R} K \subset K \otimes \mathcal{A}, \tilde{\Delta} K=0$. Therefore $K \subset$ ker $\tilde{\Delta}$ is a bicovariant subbimodule of $\dot{\Gamma} \otimes_{\mathcal{A}} \dot{\Gamma}$ (see Lemma 1.2). It remains to prove ker $\tilde{\Delta}=\operatorname{ker}(\rho+$ id). Let $x=a_{i j} \dot{p}_{i} \otimes \dot{p}_{j}$. If $x \in \operatorname{ker} \tilde{\Delta}$ then

$$
\begin{gathered}
0=\tilde{\Delta} a_{i j} \dot{p_{i}} \otimes \dot{p_{j}}=\Delta\left(a_{i j}\right)\left[\left(\dot{p}_{i} \otimes I\right)\left(\Lambda_{j s} \otimes \dot{p_{s}}\right)+\left(\Lambda_{i s} \otimes \dot{p_{s}}\right)\left(\dot{p_{j}} \otimes I\right)\right]_{\dot{\Gamma} \otimes \dot{\Gamma}}= \\
\Delta\left(a_{i j}\right)\left\{\left[\left(\Lambda_{j s} * f_{i m}\right)+\Lambda_{i s} \delta_{j m}\right] \otimes I\right\} \dot{p}_{m} \otimes \dot{p_{s}} .
\end{gathered}
$$

Using the independence of $\dot{p}_{i}$ and acting by $\epsilon \otimes \mathrm{id}$, one gets

$$
a_{i j}\left[f_{i m}\left(\Lambda_{j s}\right)+\delta_{i s} \delta_{j m}\right]=0 .
$$

Multiplying from the right by $\dot{p_{s}} \otimes p_{m}^{*}$ and using (1.13), we obtain $(\rho+\mathrm{id})(x)=$ $(\rho+\mathrm{id})\left(a_{i j} \dot{p}_{i} \otimes \dot{p}_{j}\right)=0$, i.e. $x \in \operatorname{ker}(\rho+\mathrm{id})$. Conversely, the last equality implies $(1.20)$. Acting by $\Delta$ and multiplying from the right by $\left(\Lambda_{s n} \otimes I\right)\left(p_{m} \otimes \dot{p_{n}}\right)$, we can get back $\tilde{\Delta} a_{i j} \dot{p}_{i} \otimes \dot{p_{j}}=0, x \in \operatorname{ker} \tilde{\Delta}$.

We know that (1.14) holds for some $a_{i j}^{\alpha} \in \mathrm{C}$. Then $a_{i j}^{\alpha}\left(\dot{p}_{i} \otimes \dot{p}_{j}\right)(\alpha=$ $1, \ldots, N)$ form a basis of the left module $K$. Let

$$
s^{\alpha}=a_{i j}^{\alpha} \tilde{p}_{i} \tilde{p}_{j}+b_{i}^{\alpha} \tilde{p}_{i}+c^{\alpha}
$$

be the corresponding basis elements of the left module $J_{2}\left(J_{2} \cap \tilde{\mathcal{B}}^{1}=\{0\}\right)$. We get

Proposition 1.6 As left module $\mathcal{A} \cdot \operatorname{span}\left\{p_{i} p_{j}, p_{i}, I: i, j \in \mathcal{I}\right\} \approx \tilde{\mathcal{B}}^{2} / J_{2}$ has dimension $|\mathcal{I}|^{2}+|\mathcal{I}|+1-\operatorname{dim} K$. 


\section{Properties of inhomogeneous quantum groups}

Here we continue the investigations of the previous Section (assuming the conditions given at its beginning) and find the form of commutation relations in $\mathcal{B}$. As before $\operatorname{Poly}(G)=(\mathcal{B}, \Delta)$ and $\operatorname{Poly}(H)=(\mathcal{A}, \Delta)$. Moreover, we assume

a. Each representation of $H$ is completely reducible

b. $\Lambda$ is an irreducible representation of $H$

c. $\operatorname{Mor}(v \oplus w, \Lambda \oplus v \oplus w)=\{0\}$ for any two irreducible representations $v, w$ of $H$.

The condition c. is used only for simplicity and will be removed in Remark 3.7 .

We return to the investigation of (1.9). Due to the condition a., $a=u_{A B}$, $u \in \operatorname{Irr} H, A, B=1, \ldots, \operatorname{dim} u$, form a basis of $\mathcal{A}$. Setting $\phi_{s}\left(u_{A B}\right)=\phi_{s A, B}$, (1.9) is equivalent to

$$
\Delta \phi_{s A, B}=(\Lambda \oplus u)_{s A, t C} \otimes \phi_{t C, B}+\phi_{s A, C} \otimes u_{C B} .
$$

Multiplying both sides from the right by $\Delta\left(u_{D B}^{c}\right)=u_{D L}^{c} \otimes u_{L B}^{c}$ (where $u_{D B}^{c}=$ $u_{B D}^{-1}$ etc.) and setting $\rho_{s A D}=\phi_{s A, B} u_{B D}^{-1}$, one gets

$$
\Delta \rho_{s A D}=\left(\Lambda \oplus u \oplus u^{c}\right)_{s A D, t C L} \otimes \rho_{t C L}+\rho_{s A D} \otimes I .
$$

Therefore $\left(\begin{array}{cc}\Lambda \oplus u \oplus u^{c} & \rho \\ 0 & I\end{array}\right)$ is a representation of $H$. Using the condition a., there must exist a vector $\left(\begin{array}{c}w \\ 1\end{array}\right)$ corresponding to the representation $I$. It means

$$
\rho=w-\left(\Lambda \oplus u \oplus u^{c}\right) w
$$

(conversely, (2.2) implies (2.1)). We define $\eta_{i} \in \mathcal{A}^{\prime}$ by $\eta_{i}\left(u_{A B}\right)=w_{i A B}$. Using (2.2), we get

$$
\phi_{s}\left(u_{A B}\right)=\phi_{s A, B}=\rho_{s A D} u_{D B}=\eta_{s}\left(u_{A D}\right) u_{D B}-(\Lambda \oplus u)_{s A, t L} \eta_{t}\left(u_{L B}\right)
$$

and $(1.9)$ is equivalent to

$$
\phi_{s}(a)=a * \eta_{s}-\Lambda_{s t}\left(\eta_{t} * a\right), \quad a \in \mathcal{A} .
$$


Due to c. $(v=u, w=I), \eta_{s}$ are uniquely determined by $\phi_{s}$. Inserting (2.3) to (1.7), we obtain $\eta_{s}(I)=0$ and

$$
\begin{gathered}
a b * \eta_{s}-\Lambda_{s m}\left(\eta_{m} * a b\right)= \\
\left(a * f_{s t}\right)\left(b * \eta_{t}\right)-\left(a * f_{s t}\right) \Lambda_{t m}\left(\eta_{m} * b\right)+\left(a * \eta_{s}\right) b-\Lambda_{s t}\left(\eta_{t} * a\right) b,
\end{gathered}
$$

which (see (1.5)) we can write as

$$
\left.\begin{array}{l}
a b * \eta_{s}-\left(a * f_{s t}\right)\left(b * \eta_{t}\right)-\left(a * \eta_{s}\right) b= \\
\Lambda_{s m}\left[\eta_{m} * a b-\left(f_{m t} * a\right)\left(\eta_{t} * b\right)-\left(\eta_{m} * a\right) b\right] .
\end{array}\right\}
$$

Setting $L_{m A B, C D}^{v w}=\eta_{m}(a b)-f_{m t}(a) \eta_{t}(b)-\eta_{m}(a) \epsilon(b)$ for $a=v_{A C}, b=w_{B D}$, $v, w \in \operatorname{Irr} H$, we can replace (2.4) by $L^{v w} \in \operatorname{Mor}(v \oplus w, \Lambda \oplus v \oplus w)=\{0\}$, so (1.9) is equivalent to

$$
\eta_{m}(a b)=\eta_{m}(a) \epsilon(b)+f_{m t}(a) \eta_{t}(b), \quad a, b \in \mathcal{A}, \quad \eta_{m}(I)=0 .
$$

Combining (1.3) with (2.5),

$$
\mathcal{A} \ni a \longrightarrow \rho(a)=\left(\begin{array}{cc}
f(a) & \eta(a) \\
0 & \epsilon(a)
\end{array}\right) \in M_{|\mathcal{I}|}(\mathbf{C}) \text { is a unital homomorphism. }
$$

We get

Theorem 2.1 Let $\mathcal{A}$ be a Hopf algebra satisfying a.-c.. Then the general bialgebra $\mathcal{B}$ satisfying the conditions $1 .-5$. is equal $\tilde{\mathcal{B}} / J$ where $\tilde{\mathcal{B}}$ is the algebra with $I$ generated by $\mathcal{A}$ and $\tilde{p}_{s}(s \in \mathcal{I})$ with relations (1.0) where $\phi_{s}$ is given by (2.3) for $f$ and $\eta$ satisfying (1.5) and (2.0). Moreover, $\tilde{\mathcal{B}}$ is a bialgebra with comultiplication given by the comultiplication in $\mathcal{A}$ and (1.1). $J$ is an ideal in $\tilde{\mathcal{B}}$ such that $\Delta J \subset J \otimes \tilde{\mathcal{B}}+\tilde{\mathcal{B}} \otimes J, \epsilon(J)=0, J \cap \tilde{\mathcal{B}}^{1}=\{0\}$. Conversely, each such $f, \eta$ and $J$ give $\mathcal{B}=\tilde{\mathcal{B}} / J$ satisfying the conditions $1 .-5$.

Proof. It follows from the previous considerations. 
Let us recall that $s^{\alpha}, \alpha=1, \ldots, N=\operatorname{dim} K$, form a basis of the left module $J_{2}=J \cap \tilde{\mathcal{B}}^{2}$. Due to (1.21) and (1.10),

$$
\begin{gathered}
\Delta s^{\alpha}=a_{i j}^{\alpha} \Lambda_{i m} \Lambda_{j n} \otimes \tilde{p}_{m} \tilde{p}_{n}+a_{i j}^{\alpha} \tilde{p}_{i} \tilde{p}_{j} \otimes I+ \\
a_{i j}^{\alpha}\left(\Lambda_{i m} \otimes \tilde{p}_{m}\right)\left(\tilde{p}_{j} \otimes I\right)+a_{i j}^{\alpha}\left(\tilde{p}_{i} \otimes I\right)\left(\Lambda_{j n} \otimes \tilde{p}_{n}\right)+ \\
\Delta\left(b_{i}^{\alpha}\right)\left(\Lambda_{i j} \otimes \tilde{p}_{j}\right)+\Delta\left(b_{i}^{\alpha}\right)\left(\tilde{p}_{i} \otimes I\right)+\Delta\left(c^{\alpha}\right) \in \\
\mathcal{A} \otimes J_{2} \oplus J_{2} \otimes \mathcal{A} .
\end{gathered}
$$

In particular, the terms in $\tilde{\Gamma} \otimes \tilde{\Gamma}$ should cancel out, which is equivalent (cf the proof of Proposition 1.4) to (1.20) for $a=a^{\alpha}$, i.e. to (1.19). The equations (1.19) are down to earth formulation of the condition $K \subset \operatorname{ker}(\rho+\mathrm{id})$. Using that and (1.15), one gets

$$
\Delta s^{\alpha}-s^{\alpha} \otimes I-g_{\alpha \beta} \otimes s^{\beta} \in\left(\mathcal{A} \otimes J_{2} \oplus J_{2} \otimes \mathcal{A}\right) \cap\left(\mathcal{A} \otimes \tilde{\mathcal{B}}^{1}+\tilde{\mathcal{B}}^{1} \otimes \mathcal{A}\right)=\{0\} .
$$

Thus (2.7) is equivalent to

$$
\begin{gathered}
g_{\alpha \beta} \otimes\left[-b_{i}^{\beta} \tilde{p}_{i}-c^{\beta}\right]+\left[-b_{i}^{\alpha} \tilde{p}_{i}-c^{\alpha}\right] \otimes I+ \\
a_{i j}^{\alpha} \phi_{i}\left(\Lambda_{j n}\right) \otimes \tilde{p}_{n}+\Delta\left(b_{i}^{\alpha}\right)\left(\Lambda_{i j} \otimes \tilde{p}_{j}\right)+ \\
\Delta\left(b_{i}^{\alpha}\right)\left(\tilde{p}_{i} \otimes I\right)+\Delta\left(c^{\alpha}\right)=0 .
\end{gathered}
$$

Using Lemma 1.1,

$$
\begin{gathered}
\Delta\left(b_{i}^{\alpha}\right)=b_{i}^{\alpha} \otimes I, \\
\Delta\left(b_{i}^{\alpha}\right)\left\{\left[\Lambda_{i j}-g_{\alpha \beta} b_{j}^{\beta}+a_{i k}^{\alpha} \phi_{i}\left(\Lambda_{k j}\right)\right] \otimes I\right\}=0, \\
\Delta\left(c^{\alpha}\right)=g_{\alpha \beta} \otimes c^{\beta}+c^{\alpha} \otimes I .
\end{gathered}
$$

In virtue of (2.8), $b_{i}^{\alpha} \in \mathbf{C}$. Using (2.3) and (1.15), we can write (2.9) as

$$
\left[b_{s}^{\alpha}+a_{i k}^{\alpha} \eta_{i}\left(\Lambda_{k s}\right)\right] \Lambda_{s j}=g_{\alpha \beta}\left[b_{j}^{\beta}+a_{m r}^{\beta} \eta_{m}\left(\Lambda_{r j}\right)\right] .
$$

Using the condition c. for $v=\Lambda, w=I$ (according to the condition a., $g$ is equivalent to a subrepresentation of $\Lambda \oplus \Lambda)$, we get $\operatorname{Mor}(\Lambda, g)=\{0\}$ and hence 2.9 ) is equivalent to

$$
b_{s}^{\alpha}=-a_{i k}^{\alpha} \eta_{i}\left(\Lambda_{k s}\right)
$$


Decomposing $g$ into a direct sum of irreducible representations, $c^{\alpha}$ has also a decomposition into a direct sum. So we can solve (2.10) in each irreducible component and thus assume that $g$ is irreducible. For any $v \in \operatorname{Irr} H$ we set $\mathcal{A}_{v}=\operatorname{span}\left\{v_{m n}: m, n=1, \ldots, \operatorname{dim} v\right\}$. We know $\mathcal{A}=\oplus_{v \in \operatorname{Irr}}{ }_{H} \mathcal{A}_{v}$.

i) $g=I$, hence $\Delta\left(c^{\alpha}\right)=I \otimes c^{\alpha}+c^{\alpha} \otimes I$. Thus

$$
\begin{gathered}
\Delta c^{\alpha} \in\left(\oplus_{v \in \operatorname{Irr} H} \mathcal{A}_{v} \otimes \mathcal{A}_{v}\right) \cap\left(\oplus_{v \in \operatorname{Irr} H}\left(\mathcal{A}_{I} \otimes \mathcal{A}_{v} \oplus \mathcal{A}_{v} \otimes \mathcal{A}_{I}\right)\right)= \\
\mathcal{A}_{I} \otimes \mathcal{A}_{I}=\mathbf{C} I \otimes I,
\end{gathered}
$$

$c^{\alpha}=\lambda I, \lambda \in \mathbf{C}$. That gives $\lambda=2 \lambda, \lambda=0, c^{\alpha}=0$.

ii) $g \in \operatorname{Irr} H, g \not f I$. Then $c^{\alpha}=\sum c_{v}^{\alpha}, c_{v}^{\alpha} \in \mathcal{A}_{v}$ and (2.10) is equivalent to

$$
\begin{gathered}
\Delta c_{I}^{\alpha}=c_{I}^{\alpha} \otimes I \in \mathcal{A}_{I} \otimes \mathcal{A}_{I}, \\
0=g_{\alpha \beta} \otimes c_{I}^{\beta}+c_{g}^{\alpha} \otimes I \in \mathcal{A}_{g} \otimes \mathcal{A}_{I}, \\
\Delta c_{g}^{\alpha}=g_{\alpha \beta} \otimes c_{g}^{\beta} \in \mathcal{A}_{g} \otimes \mathcal{A}_{g}, \\
\Delta c_{v}^{\alpha}=0 \in \mathcal{A}_{v} \otimes \mathcal{A}_{v}, \quad 0=g_{\alpha \beta} \otimes c_{v}^{\beta} \in \mathcal{A}_{g} \otimes \mathcal{A}_{v}, \\
0=c_{v}^{\alpha} \otimes I \in \mathcal{A}_{v} \otimes \mathcal{A}_{I}, \quad v \in \operatorname{Irr} H, \quad v \not \sim I, g .
\end{gathered}
$$

Solving these relations, one gets $c_{I}^{\alpha} \in \mathbf{C}, c_{g}^{\alpha}=-g_{\alpha \beta} c_{I}^{\beta}, c_{v}^{\alpha}=0$ for $v \neq I, g$, $v \in \operatorname{Irr} H$. Then $c^{\alpha}=c_{I}^{\alpha}-g_{\alpha \beta} c_{I}^{\beta}$. It holds also in the case i) and for whole $g$.

Since $a^{\alpha}$ are linearly independent, there exist $T_{m n} \in \mathbf{C}$ such that $c_{I}^{\alpha}=$ $a_{m n}^{\alpha} T_{m n}$. One gets $c^{\alpha}=a_{m n}^{\alpha}\left(T_{m n}-\Lambda_{m a} \Lambda_{n b} T_{a b}\right)$ (we have used (1.15)). Concluding,

$$
s^{\alpha}=a_{i j}^{\alpha}\left(\tilde{p}_{i} \tilde{p}_{j}-\eta_{i}\left(\Lambda_{j s}\right) \tilde{p}_{s}+T_{i j}-\Lambda_{i m} \Lambda_{j n} T_{m n}\right)
$$

and we get $(N=\operatorname{dim} K)$

Theorem 2.2 Let $\mathcal{B}$ be as in Theorem 2.1. Then $J_{2}=J \cap \tilde{\mathcal{B}}^{2}$ is an $\mathcal{A}$ bimodule and as the left module it has a basis (2.19), $\alpha=1, \ldots, N$, for some $a_{i j}^{\alpha}, T_{i j} \in \mathbf{C}, N \in \mathbf{N}$. Moreover, $a^{\alpha}$ satisfy (1.19), (1.15) and (1.10)-(1.17) for some $g \in \operatorname{Rep} H$ and $\omega_{\alpha \beta} \in \mathcal{A}^{\prime}(\alpha, \beta=1, \ldots, N)$. 
Theorem 2.3 Let $j_{2} \subset \tilde{\mathcal{B}}^{2}$ be the left module generated by (2.19) for some $a_{i j}^{\alpha}, T_{i j} \in \mathbf{C}$, such that $a^{\alpha}(\alpha=1, \ldots, N)$ are linearly independent and satisfy (1.19), 1.15) and (1.10)-1.17) for some $g \in \operatorname{Rep} H$ and $\omega_{\alpha \beta} \in \mathcal{A}^{\prime}$. Then

$$
\Delta j_{2} \subset\left(j_{2} \otimes \mathcal{A}\right) \oplus\left(\mathcal{A} \otimes j_{2}\right), \quad j_{2} \cap \tilde{\mathcal{B}}^{1}=\{0\} .
$$

Moreover, $j_{2}$ is a bimodule if and only if

$$
g_{\alpha \beta}\left(\tau_{\beta} * b\right)=b * \tau_{\alpha}, \quad b \in \mathcal{A},
$$

where $\tau_{\alpha}=a_{i j}^{\alpha} \tau_{i j}$,

$$
\tau_{i j}=\eta_{j} * \eta_{i}-\eta_{i}\left(\Lambda_{j s}\right) \eta_{s}+T_{i j} \epsilon-\left(f_{j n} * f_{i m}\right) T_{m n}
$$

and $g$ is given by (1.15).

Proof. The first statement follows from the computations before Theorem 2.2. Due to Proposition [1.3, $j_{2} / \tilde{\mathcal{B}}^{1}$ is a bicovariant bimodule contained in $\operatorname{ker}(\rho+\mathrm{id})$. In order to prove the last statement we compute

$$
\begin{aligned}
& s^{\alpha} b=a_{i j}^{\alpha}\left[\tilde{p}_{i} \tilde{p}_{j}-\eta_{i}\left(\Lambda_{j s}\right) \tilde{p}_{s}+T_{i j}-\Lambda_{i m} \Lambda_{j n} T_{m n}\right] b= \\
& a_{i j}^{\alpha}\left\{\tilde{p}_{i}\left[\left(b * f_{j s}\right) \tilde{p}_{s}+\phi_{j}(b)\right]-\eta_{i}\left(\Lambda_{j s}\right) \tilde{p}_{s} b+\left(T_{i j}-\Lambda_{i m} \Lambda_{j n} T_{m n}\right) b\right\}= \\
& a_{i j}^{\alpha}\left\{\left(b * f_{j s} * f_{i m}\right) \tilde{p}_{m} \tilde{p}_{s}+\phi_{i}\left(b * f_{j r}\right) \tilde{p}_{r}+\left[\phi_{j}(b) * f_{i r}\right] \tilde{p}_{r}+\right. \\
& \phi_{i}\left(\phi_{j}(b)\right)-\eta_{i}\left(\Lambda_{j s}\right)\left(b * f_{s r}\right) \tilde{p}_{r}-\eta_{i}\left(\Lambda_{j s}\right) \phi_{s}(b)+ \\
& \left.\left(T_{i j}-\Lambda_{i m} \Lambda_{j n} T_{m n}\right) b\right\} .
\end{aligned}
$$

Using (1.16), we get

$$
\left(b * \xi_{\alpha \beta}\right) s_{\beta}=a_{i j}^{\alpha}\left(b * f_{j n} * f_{i m}\right)\left(\tilde{p}_{m} \tilde{p}_{n}-\eta_{m}\left(\Lambda_{n r}\right) \tilde{p}_{r}+T_{m n}-\Lambda_{m a} \Lambda_{n b} T_{a b}\right),
$$

hence

$$
s^{\alpha} b-\left(b * \xi_{\alpha \beta}\right) s^{\beta}=A_{\alpha r} \tilde{p}_{r}+B_{\alpha}
$$

for some $A_{\alpha r}, B_{\alpha} \in \mathcal{A}$. We conclude that $j_{2}$ is a right module if and only if (2.17) belongs to $j_{2}$ for any $b$, which means $A_{\alpha r}=B_{\alpha}=0\left(j_{2} \cap \tilde{\mathcal{B}}^{1}=\{0\}\right)$. 
Using (2.15), (2.16), (2.17), (2.3) and (1.3), one obtains

$$
\begin{gathered}
A_{\alpha r}=a_{i j}^{\alpha}\left\{b * f_{j r} * \eta_{i}-\Lambda_{i m}\left(\eta_{m} * b * f_{j r}\right)+\right. \\
b * \eta_{j} * f_{i r}-\left(\Lambda_{j s} * f_{i m}\right)\left(\eta_{s} * b * f_{m r}\right)- \\
\left.\eta_{i}\left(\Lambda_{j s}\right)\left(b * f_{s r}\right)+\left(b * f_{j n} * f_{i m}\right) \eta_{m}\left(\Lambda_{n r}\right)\right\} .
\end{gathered}
$$

In virtue of (1.19)

$$
\begin{gathered}
a_{i j}^{\alpha}\left(\Lambda_{j s} * f_{i m}\right)=a_{i j}^{\alpha} f_{i m}\left(\Lambda_{j k}\right) \Lambda_{k s}= \\
a_{i j}^{\alpha} R_{i j, k m} \Lambda_{k s}=-a_{k m}^{\alpha} \Lambda_{k s}
\end{gathered}
$$

so the second and the fourth terms in $A_{\alpha r}$ cancel. On the other hand, (1.5), (2.5) imply

$$
\left.\begin{array}{c}
0=\eta_{i}\left\{\left(b * f_{j s}\right) \Lambda_{s r}-\Lambda_{j s}\left(f_{s r} * b\right)\right\}= \\
\eta_{i}\left(b * f_{j r}\right)+f_{i m}\left(b * f_{j s}\right) \eta_{m}\left(\Lambda_{s r}\right)- \\
\eta_{i}\left(\Lambda_{j s}\right) f_{s r}(b)-f_{i m}\left(\Lambda_{j s}\right) \eta_{m}\left(f_{s r} * b\right),
\end{array}\right\}
$$

hence due to (1.19)

$$
\left.\begin{array}{c}
a_{i j}^{\alpha}\left[\left(f_{j r} * \eta_{i}\right)(b)+\left(f_{j s} * f_{i m}\right)(b) \eta_{m}\left(\Lambda_{s r}\right)-\right. \\
\left.\eta_{i}\left(\Lambda_{j s}\right) f_{s r}(b)+\left(\eta_{j} * f_{i r}\right)(b)\right]=0 .
\end{array}\right\}
$$

Therefore also other terms in $A_{\alpha r}$ vanish, $A_{\alpha r}=0$ for each $b \in \mathcal{A}$.

In virtue of (2.3), (1.7) and (1.5),

$$
\begin{gathered}
B_{\alpha}=a_{i j}^{\alpha}\left[\phi_{i}\left(b * \eta_{j}\right)-\left(\Lambda_{j m} * f_{i s}\right) \phi_{s}\left(\eta_{m} * b\right)-\right. \\
\phi_{i}\left(\Lambda_{j s}\right)\left(\eta_{s} * b\right)-\eta_{i}\left(\Lambda_{j s}\right) \phi_{s}(b)+\left(T_{i j}-\Lambda_{i m} \Lambda_{j n} T_{m n}\right) b- \\
\left.\left(b * f_{j n} * f_{i m}\right)\left(T_{m n}-\Lambda_{m a} \Lambda_{n b} T_{a b}\right)\right]= \\
a_{i j}^{\alpha} \cdot\left[b * \eta_{j} * \eta_{i}-\Lambda_{i m}\left(\eta_{m} * b * \eta_{j}\right)-\right. \\
f_{i s}\left(\Lambda_{j l}\right) \Lambda_{l m}\left(\eta_{m} * b * \eta_{s}\right)+f_{i s}\left(\Lambda_{j l}\right) \Lambda_{l m} \Lambda_{s n}\left(\eta_{n} * \eta_{m} * b\right)- \\
\eta_{i}\left(\Lambda_{j m}\right) \Lambda_{m s}\left(\eta_{s} * b\right)+\Lambda_{i m} \Lambda_{j n} \eta_{m}\left(\Lambda_{n s}\right)\left(\eta_{s} * b\right)- \\
\eta_{i}\left(\Lambda_{j s}\right)\left(b * \eta_{s}\right)+\eta_{i}\left(\Lambda_{j s}\right) \Lambda_{s m}\left(\eta_{m} * b\right)+
\end{gathered}
$$




$$
\begin{gathered}
T_{i j} b-\Lambda_{i m} \Lambda_{j n} T_{m n} b-\left(b * f_{j n} * f_{i m}\right) T_{m n}+ \\
\left.\Lambda_{i m} \Lambda_{j n}\left(f_{n c} * f_{m a} * b\right) T_{a c}\right] .
\end{gathered}
$$

The fifth and the eight terms cancel. Using (11.19), the second and the third terms also give 0 . The terms $1,7,9$ and 11 produce $b * \tau_{\alpha}$. In virtue of $a_{i j}^{\alpha} \Lambda_{i m} \Lambda_{j n}=g_{\alpha \beta} a_{m n}^{\beta}$ (see (1.15)) and (1.19) the terms 4,6,10 and 12 yield $-g_{\alpha \beta}\left(\tau_{\beta} * b\right)$. Thus $B_{\alpha}=b * \tau_{\alpha}-g_{\alpha \beta}\left(\tau_{\beta} * b\right)$ and our Theorem follows.

Using the notation of Theorem 2.3 one has

\section{Proposition 2.4}

$$
\tau_{\alpha}(a b)=\omega_{\alpha \beta}(a) \tau_{\beta}(b)+\tau_{\alpha}(a) \epsilon(b) \quad(a, b \in \mathcal{A}), \quad \tau_{\alpha}(I)=0 .
$$

Proof. We have (see (1.3), (2.5))

$$
\begin{gathered}
\tau_{i j}(a b)=\left(\eta_{j} * \eta_{i}\right)(a b)-\eta_{i}\left(\Lambda_{j s}\right)\left[f_{s r}(a) \eta_{r}(b)+\eta_{s}(a) \epsilon(b)\right]+ \\
T_{i j} \epsilon(a) \epsilon(b)-\left(f_{j s} * f_{i m}\right)(a)\left(f_{s r} * f_{m l}\right)(b) T_{l r} .
\end{gathered}
$$

But (we use (2.5), (2.19) and (1.16))

$$
\begin{gathered}
a_{i j}^{\alpha}\left(\eta_{j} * \eta_{i}\right)(a b)=a_{i j}^{\alpha}\left[\left(\eta_{j} * \eta_{i}\right)(a) \epsilon(b)+\left(f_{j r} * \eta_{i}\right)(a) \eta_{r}(b)+\right. \\
\left.\left(\eta_{j} * f_{i r}\right)(a) \eta_{r}(b)+\left(f_{j r} * f_{i s}\right)(a)\left(\eta_{r} * \eta_{s}\right)(b)\right]= \\
a_{i j}^{\alpha}\left[\left(\eta_{j} * \eta_{i}\right)(a) \epsilon(b)+\eta_{i}\left(\Lambda_{j s}\right) f_{s r}(a) \eta_{r}(b)-\right. \\
\left.\left(f_{j s} * f_{i m}\right)(a) \eta_{m}\left(\Lambda_{s r}\right) \eta_{r}(b)+\left(f_{j s} * f_{i m}\right)(a)\left(\eta_{s} * \eta_{m}\right)(b)\right]= \\
a_{i j}^{\alpha}\left[\left(\eta_{j} * \eta_{i}\right)(a) \epsilon(b)+\eta_{i}\left(\Lambda_{j s}\right) f_{s r}(a) \eta_{r}(b)\right]+ \\
\omega_{\alpha \beta}(a) a_{m s}^{\beta}\left[\left(\eta_{s} * \eta_{m}\right)(b)-\eta_{m}\left(\Lambda_{s r}\right) \eta_{r}(b)\right] .
\end{gathered}
$$

Combining these facts, we get the first assertion. The second one is trivial.๑.

Remark 2.5 Proposition 2.4 and (1.17) give that

$$
\zeta: \mathcal{A} \ni a \longrightarrow\left(\begin{array}{cc}
\omega(a) & \tau(a) \\
0 & \epsilon(a)
\end{array}\right) \in M_{N+1}(\mathbf{C})
$$

is a unital homomorphism, where $\omega(a)=\left(\omega_{\alpha \beta}(a)\right)_{\alpha, \beta=1}^{N}, \tau(a)=\left(\tau_{\alpha}(a)\right)_{\alpha=1}^{N}$. 
Remark 2.6 Let $S$ be a set generating $\mathcal{A}$ as algebra with unity. One can prove that (2.19) for $b \in S$ implies (2.19) for $b \in \mathcal{A}$ (due to (2.17) $A_{\alpha r}=0$ for $b, b^{\prime}$ implies $A_{\alpha r}=0$ for $\left.b b^{\prime}\right)$. Similarly, (2.14) for $b \in S$ implies (2.14) for $b \in \mathcal{A}$ (it is equivalent to the right module condition which suffices to check only for $b \in S$ ).

\section{Structure of inhomogeneous quantum groups}

Here we continue the investigations of two preceding Sections (including the assumptions made at their beginnings) and find the exact form and 'size' of inhomogenous quantum groups. From now on we shall consider the most natural situation (which is the case for quantum Poincaré groups):

$$
R^{2}=\mathbf{1}^{\otimes 2} \quad \text { and } \quad x\left(R+\mathbf{1}^{\otimes 2}\right)=0 \Leftrightarrow x \in \operatorname{span}\left\{a^{\alpha}: \alpha=1, \ldots, \operatorname{dim} K\right\}
$$

$\left(\operatorname{cf}(1.11)\right.$ and (1.19)). In other words: $\rho^{2}=\mathrm{id}$ and $K=J_{2} / \tilde{\mathcal{B}}^{1}=\operatorname{ker}(\rho+\mathrm{id})$. The second condition means that we have as many relations $a_{i j}^{\alpha} p_{i} p_{j}+b_{i}^{\alpha} p_{i}+$ $c^{\alpha}=0$ as it is allowed by (1.19) (for $b_{i}^{\alpha}=c^{\alpha}=0 p_{i}$ would be R-symmetric: $\left.R_{k l, i j} p_{i} p_{j}=p_{k} p_{l}\right)$.

We set

$$
A_{3}=\mathbf{1} \otimes \mathbf{1} \otimes \mathbf{1}-R \otimes \mathbf{1}-\mathbf{1} \otimes R+(R \otimes \mathbf{1})(\mathbf{1} \otimes R)+
$$$$
(\mathbf{1} \otimes R)(R \otimes \mathbf{1})-(R \otimes \mathbf{1})(\mathbf{1} \otimes R)(R \otimes \mathbf{1}),
$$

$F_{i j k, m}=\tau_{i j}\left(\Lambda_{k m}\right), Z_{i j, m}=\eta_{i}\left(\Lambda_{j m}\right)$. The main result of the Section is contained in

Theorem 3.1 Let $f, \eta$ satisfy (1.5), (2.0) and $\rho^{2}=\mathrm{id}$. The following conditions are equivalent:

i) $J$ is as in Theorem 2.1 with $K=J_{2} / \tilde{\mathcal{B}}^{1}=\operatorname{ker}(\rho+\mathrm{id})$

ii) $J$ is the ideal generated by

$$
s_{k l}=\left(R-\mathbf{1}^{\otimes 2}\right)_{k l, i j}\left(\tilde{p}_{i} \tilde{p}_{j}-\eta_{i}\left(\Lambda_{j s}\right) \tilde{p}_{s}+T_{i j}-\Lambda_{i m} \Lambda_{j n} T_{m n}\right)
$$

for some complex numbers $\left\{T_{i j}\right\}_{i, j \in \mathcal{I}}$ satisfying (2.14),

$$
A_{3} F=0,
$$

$$
A_{3}(Z \otimes \mathbf{1}-\mathbf{1} \otimes Z) T \in \operatorname{Mor}(I, \Lambda \oplus \Lambda \oplus \Lambda) .
$$


If the condition i) or ii) is satisfied then

$$
J_{2}=\mathcal{A} \cdot \operatorname{span}\left\{s_{k l}: k, l \in \mathcal{I}\right\}
$$

and $\mathcal{B}=\tilde{\mathcal{B}} / J$ satisfies the conditions $1 .-5$.

Proof. One has

$$
R^{2}=\mathbf{1}^{\otimes 2} .
$$

Let $J$ be as in Theorem 2.1, $J_{2}=J \cap \tilde{\mathcal{B}}^{2}$ and $K=J_{2} / \tilde{\mathcal{B}}^{1}=\operatorname{ker}(\rho+i d)$. All the conditions of Proposition 1.3 are satisfied in that case. Theorem 2.2 and Theorem 2.3 give (2.14). Moreover (cf the beginning of the Section),

$$
\operatorname{span}\left\{a^{\alpha}: \alpha=1, \ldots, \operatorname{dim} K\right\}=\operatorname{span}\left\{a^{k l}: k, l=1, \ldots,|\mathcal{I}|\right\}
$$

where $\left(a^{k l}\right)_{i j}=\left(R-\mathbf{1}^{\otimes 2}\right)_{k l, i j}$. Thus (3.3) is satisfied (see Theorem 2.2). Hence, in $\mathcal{B}$

$$
p_{k} p_{l}=R_{k l, i j} p_{i} p_{j}+r_{k l}
$$

where

$$
\begin{gathered}
r_{k l}=c_{k l, s} p_{s}+M_{k l}, \\
c_{k l, s}=-\left(R-\mathbf{1}^{\otimes 2}\right)_{k l, i j} \eta_{i}\left(\Lambda_{j s}\right)
\end{gathered}
$$

(i.e. $\left.c=-\left(R-\mathbf{1}^{\otimes 2}\right) Z\right)$,

$$
\begin{gathered}
M_{k l}=\left(R-\mathbf{1}^{\otimes 2}\right)_{k l, i j}\left(T_{i j}-W_{i j}\right), \\
W_{i j}=\Lambda_{i m} \Lambda_{j n} T_{m n} .
\end{gathered}
$$

In short,

$$
p \oplus p=R(p \oplus p)+r .
$$

Therefore

$$
\begin{aligned}
& p \oplus p \oplus p=(p \oplus p) \oplus p=(R \otimes \mathbf{1})(p \oplus(p \oplus p))+r \oplus p= \\
& (R \otimes \mathbf{1})[(\mathbf{1} \otimes R)((p \oplus p) \oplus p)+p \oplus r]+r \oplus p= \\
& (R \otimes \mathbf{1})(\mathbf{1} \otimes R)(R \otimes \mathbf{1})(p \oplus p \oplus p)+(R \otimes \mathbf{1})(\mathbf{1} \otimes R)(r \oplus p)+ \\
& (R \otimes \mathbf{1})(p \oplus r)+r \oplus p .
\end{aligned}
$$


On the other hand,

$$
\begin{gathered}
p \oplus p \oplus p=p \oplus(p \oplus p)=(\mathbf{1} \otimes R)((p \oplus p) \oplus p)+p \oplus r= \\
(\mathbf{1} \otimes R)[(R \otimes \mathbf{1})(p \oplus(p \oplus p))+r \oplus p]+p \oplus r= \\
(\mathbf{1} \otimes R)(R \otimes \mathbf{1})(\mathbf{1} \otimes R)(p \oplus p \oplus p)+(\mathbf{1} \otimes R)(R \otimes \mathbf{1})(p \oplus r)+ \\
(\mathbf{1} \otimes R)(r \oplus p)+p \oplus r .
\end{gathered}
$$

But the braid equation for $\rho$ (see (3.8) of [15]) implies

$$
(R \otimes \mathbf{1})(\mathbf{1} \otimes R)(R \otimes \mathbf{1})=(\mathbf{1} \otimes R)(R \otimes \mathbf{1})(\mathbf{1} \otimes R) .
$$

Thus

$$
A(r \oplus p)=B(p \oplus r)
$$

where

$$
\begin{aligned}
& A=(R \otimes \mathbf{1})(\mathbf{1} \otimes R)-\mathbf{1} \otimes R+\mathbf{1} \otimes \mathbf{1} \otimes \mathbf{1}, \\
& B=(\mathbf{1} \otimes R)(R \otimes \mathbf{1})-R \otimes \mathbf{1}+\mathbf{1} \otimes \mathbf{1} \otimes \mathbf{1} .
\end{aligned}
$$

Using $r=c p+M$ and (1.6), we can rewrite (3.12) as

$$
H(p \oplus p)+L p+N=0,
$$

where

$$
\begin{gathered}
H=A(c \otimes \mathbf{1})-B(\mathbf{1} \otimes c), \\
L_{i j k, s}=A_{i j k, m n s} M_{m n}-B_{i j k, m n l}\left(M_{n l} * f_{m s}\right), \\
N_{i j k}=-B_{i j k, m n l} \phi_{m}\left(M_{n l}\right) .
\end{gathered}
$$

Therefore (see (3.3)),

$$
H(\tilde{p} \oplus \tilde{p})+L \tilde{p}+N=D\left[\left(R-\mathbf{1}^{\otimes 2}\right)(\tilde{p} \oplus \tilde{p})+c \tilde{p}+M\right]
$$

for some matrix $D$, which thus satisfies $H=D\left(R-\mathbf{1}^{\otimes 2}\right)$. It exists iff

$$
H\left(R+\mathbf{1}^{\otimes 2}\right)=0
$$

and can be chosen as $D=-\frac{1}{2} H$. Consequently, (3.12) is equivalent to (3.20),

$$
L=-\frac{1}{2} H c
$$


and

$$
N=-\frac{1}{2} H M
$$

Let us now consider (3.20)-(3.22) as abstract conditions for $\eta_{i}, T_{k l}$. We shall prove that 3.20 follows from the previous conditions. By virtue of (2.19) for $b=\Lambda_{k l}$,

$$
\begin{gathered}
\left(R-\mathbf{1}^{\otimes 2}\right)_{n t, i j}\left[f_{j r}\left(\Lambda_{k a}\right) \eta_{i}\left(\Lambda_{a l}\right)+f_{j s}\left(\Lambda_{k a}\right) f_{i m}\left(\Lambda_{a l}\right) \eta_{m}\left(\Lambda_{s r}\right)-\right. \\
\left.\eta_{i}\left(\Lambda_{j s}\right) f_{s r}\left(\Lambda_{k l}\right)+\eta_{j}\left(\Lambda_{k a}\right) f_{i r}\left(\Lambda_{a l}\right)\right]=0
\end{gathered}
$$

hence

$$
\left.\begin{array}{c}
{\left[\left(R-\mathbf{1}^{\otimes 2}\right) \otimes \mathbf{1}\right]\{(\mathbf{1} \otimes R)(Z \otimes \mathbf{1})+(\mathbf{1} \otimes R)(R \otimes \mathbf{1})(\mathbf{1} \otimes Z)-} \\
(Z \otimes \mathbf{1}) R+(\mathbf{1} \otimes Z) R\}=0 .
\end{array}\right\}
$$

Multiplying from the left by $A$ and using

$$
\begin{gathered}
A\left(\left(\mathbf{1}^{\otimes 2}-R\right) \otimes \mathbf{1}\right)=B\left(\mathbf{1} \otimes\left(\mathbf{1}^{\otimes 2}-R\right)\right)=A_{3}, \\
A_{3}(\mathbf{1} \otimes R)=-A_{3}, \quad A_{3}(R \otimes \mathbf{1})=-A_{3}
\end{gathered}
$$

(it follows from (3.4) and (3.11)), we get

$$
A_{3}(\mathbf{1} \otimes Z-Z \otimes \mathbf{1})\left(\mathbf{1}^{\otimes 2}+R\right)=0 .
$$

But in virtue of (3.16) and (3.24)

$$
H=A_{3}(Z \otimes \mathbf{1}-\mathbf{1} \otimes Z)
$$

and (3.20) follows.

Now we shall consider (3.21). One has

$$
\begin{gathered}
W_{i j} * f_{m s}=\left(\Lambda_{i a} \Lambda_{j b} T_{a b}\right) * f_{m s}= \\
f_{m n}\left(\Lambda_{i c}\right) f_{n s}\left(\Lambda_{j d}\right) \Lambda_{c a} \Lambda_{d b} T_{a b}=[(R \otimes \mathbf{1})(\mathbf{1} \otimes R)(W \otimes \mathbf{1})]_{m i j, s}, \\
T_{i j} * f_{m s}=\delta_{m s} T_{i j}=(\mathbf{1} \otimes T)_{m i j, s},
\end{gathered}
$$

hence (see (3.17), (3.8), (3.24))

$$
L=A_{3}((W-T) \otimes \mathbf{1})-A_{3}(W \otimes \mathbf{1})+A_{3}(\mathbf{1} \otimes T)=A_{3}(\mathbf{1} \otimes T-T \otimes \mathbf{1}) .
$$


Using (3.20) and (3.27),

$$
\frac{1}{2} H c=H Z=A_{3}(Z \otimes \mathbf{1}-\mathbf{1} \otimes Z) Z .
$$

Moreover,

$$
\begin{gathered}
F_{i j k, m}=\tau_{i j}\left(\Lambda_{k m}\right)=\eta_{j}\left(\Lambda_{k s}\right) \eta_{i}\left(\Lambda_{s m}\right)- \\
\eta_{i}\left(\Lambda_{j s}\right) \eta_{s}\left(\Lambda_{k m}\right)+T_{i j} \delta_{k m}-f_{j n}\left(\Lambda_{k s}\right) f_{i r}\left(\Lambda_{s m}\right) T_{r n}
\end{gathered}
$$

Thus

$$
\begin{gathered}
F=(\mathbf{1} \otimes Z) Z-(Z \otimes \mathbf{1}) Z+T \otimes \mathbf{1}-(\mathbf{1} \otimes R)(R \otimes \mathbf{1})(\mathbf{1} \otimes T), \\
-A_{3} F=\frac{1}{2} H c+L,
\end{gathered}
$$

and (3.21) is equivalent to (3.1).

Finally, we investigate (3.22). According to (1.7) and (2.3),

$$
\begin{gathered}
\phi_{m}\left(W_{i j}\right)=\left(\Lambda_{i a} * f_{m s}\right) \phi_{s}\left(\Lambda_{j b}\right) T_{a b}+\phi_{m}\left(\Lambda_{i a}\right) \Lambda_{j b} T_{a b} \\
\phi_{s}\left(\Lambda_{j b}\right)=\eta_{s}\left(\Lambda_{j c}\right) \Lambda_{c b}-\Lambda_{s r} \Lambda_{j k} \eta_{r}\left(\Lambda_{k b}\right)
\end{gathered}
$$

Setting $X_{m i j}=\phi_{m}\left(W_{i j}\right)$, one gets

$X=(R \otimes \mathbf{1})(\mathbf{1} \otimes Z) W+(Z \otimes \mathbf{1}) W-(R \otimes \mathbf{1})(\Lambda \oplus \Lambda \oplus \Lambda)(\mathbf{1} \otimes Z) T-(\Lambda \oplus \Lambda \oplus \Lambda)(Z \otimes \mathbf{1}) T$.

Using (3.18), (3.8), (3.24) and (1.12), we obtain

$$
N=-A_{3} X=-A_{3}(Z \otimes \mathbf{1}-\mathbf{1} \otimes Z) W+(\Lambda \oplus \Lambda \oplus \Lambda) A_{3}(Z \otimes \mathbf{1}-\mathbf{1} \otimes Z) T .
$$

Due to 3.20 and 3.27$)$

$$
\frac{1}{2} H M=A_{3}(Z \otimes \mathbf{1}-\mathbf{1} \otimes Z)(W-T) .
$$

Therefore

$$
N+\frac{1}{2} H M=(\Lambda \oplus \Lambda \oplus \Lambda) m-m,
$$

where $m=A_{3}(Z \otimes \mathbf{1}-\mathbf{1} \otimes Z) T$ and (3.22) is equivalent to (3.2). Thus the condition i) implies (3.3), (2.14), (3.1) and (3.2) for some complex numbers $T_{i j}(i, j \in \mathcal{I})$. 
Let us now assume (2.14), (3.1) and (3.2). We define $j_{2}$ as the right hand side of (3.3). Thus $j_{2} / \mathcal{B}^{1}=K=\operatorname{ker}(\rho+\mathrm{id})$. In virtue of Proposition 1.3 and Theorem 2.3 we get (2.13) and the bimodule property of $j_{2}$. Let $j$ be the ideal generated by $j_{2}$. Then $\Delta j \subset j \otimes \tilde{\mathcal{B}}+\tilde{\mathcal{B}} \otimes j$. Moreover, $\Delta \tilde{p}=\tilde{p} \oplus I+\Lambda \oplus \tilde{p}$ implies $e(\tilde{p})=0$, hence $e\left(j_{2}\right)=0, e(j)=0$. The previous computations show that (3.12) holds in $\tilde{\mathcal{B}} / j_{2}$. We shall show $j \cap \tilde{\mathcal{B}}^{1}=\{0\}, j \cap \tilde{\mathcal{B}}^{2}=j_{2}$. Therefore $j$ is as in Theorem 2.1 and $\mathcal{B}=\tilde{\mathcal{B}} / j$ satisfies the conditions 1.-5.. Furthermore, we will prove that if $J$ satisfies the condition i) and $J \cap \tilde{\mathcal{B}}^{2}=j_{2}$ then $J=j$. Thus the proof of the Theorem will be finished.

We set $R_{k}=\mathbf{1}^{\otimes(k-1)} \otimes R \otimes \mathbf{1}^{\otimes(n-k-1)}$ (cf the notation in the Introduction), $k=1,2, \ldots, n-1, R_{\pi}=R_{i_{1}} \ldots \cdot R_{i_{s}}$ for a permutation $\pi \in \Pi_{n}$ with a minimal decomposition into transpositions

$$
\pi=t_{i_{1}} \ldots t_{i_{s}}
$$

Due to (3.11), $R_{\pi}$ is well defined. We set

$$
S_{n}=\frac{1}{n !} \sum_{\pi \in \Pi_{n}} R_{\pi}
$$

Moreover, we put $r_{n k}=p^{\oplus(k-1)} \oplus r \oplus p^{\oplus(n-k-1)}$ (see (3.6)),

$$
\left.\begin{array}{l}
r_{n \pi}=r_{n i_{1}}+R_{i_{1}} r_{n i_{2}}+R_{i_{1}} R_{i_{2}} r_{n i_{3}}+\ldots+ \\
R_{i_{1}} \cdot \ldots \cdot R_{i_{s-1}} r_{n i_{s}},
\end{array}\right\}
$$

(we choose some decomposition (3.31) for each $\pi$ ), $r_{n}=\frac{1}{n !} \sum_{\pi} r_{n \pi}$. We shall prove the following

Proposition 3.2 Let $j$ be the ideal generated by (3.3). We assume (3.19) in $\tilde{\mathcal{B}} / j_{2}$ and (2.14). Then $j$ as a left module is generated by matrix elements of

$$
\left(\mathbf{1}^{\otimes n}-S_{n}\right)\left(\tilde{p}^{\oplus n}-r_{n}\right) .
$$

Proof. In virtue of Theorem 2.3, (3.3) is an $\mathcal{A}$-bimodule and as a left module it is generated by matrix elements of $\left(\mathbf{1}^{\otimes 2}-R\right) \tilde{p}^{\oplus^{2}}-r$. Therefore $j$ is the left module generated by

$$
\left(\mathbf{1}^{\otimes m}-R_{k}\right) \tilde{p}^{\oplus m}-r_{m k},
$$


$m=2,3, \ldots, k=1, \ldots, m-1$. We set $j_{n}$ as the left module generated by (3.34) for $m=2, \ldots, n$ (for $n=2$ it coincides with the old definition of $j_{2}$ ). Thus $\mathcal{A} j_{n} \subset j_{n}, \quad j_{n} \mathcal{A} \subset j_{n}, \quad j_{n} \tilde{p}_{i} \subset j_{n+1}, \quad \tilde{p}_{i} j_{n} \subset j_{n+1}$. Moreover,

$$
\begin{gathered}
\tilde{p}^{\oplus n} \equiv R_{k} \tilde{p}^{\oplus n}+r_{n k} \quad(\bmod . j), \\
\tilde{p}^{\oplus n} \equiv R_{i_{1}}\left(R_{i_{2}} \tilde{p}^{\oplus n}+r_{n i_{2}}\right)+r_{n i_{1}} \quad(\bmod . j), \quad \text { etc. }, \\
\tilde{p}^{\oplus n} \equiv R_{\pi} \tilde{p}^{\oplus n}+r_{n \pi} \quad(\bmod . j), \quad \tilde{p}^{\oplus n} \equiv S_{n} \tilde{p}^{\oplus n}+r_{n} \quad(\bmod . j) .
\end{gathered}
$$

For any minimal decomposition (3.31) we set $r_{n \pi}^{(i)}$ as the right hand side of (3.32), where $i=\left(i_{1}, \ldots, i_{s}\right)$. We shall prove

$$
r_{n \pi}^{(i)} \equiv r_{n \pi}^{\left(i^{\prime}\right)}\left(\bmod . j_{n-1}\right)
$$

for any two such decompositions $i, i^{\prime}$. But $i, i^{\prime}$ can be obtained one from another by a finite number of steps of the following 2 kinds:

(i) we replace $\ldots t_{k} t_{l} \ldots$ by $\ldots t_{l} t_{k} \ldots$ for $|k-l|>1$,

(ii) we replace $\ldots t_{k} t_{k+1} t_{k} \ldots$ by $\ldots t_{k+1} t_{k} t_{k+1} \ldots$.

Thus it suffices to check (3.35) for each of these two cases. ad (i). We may assume $k<l-1$. One has

$$
\begin{gathered}
\left(\mathbf{1}^{\otimes n}-R_{k}\right) r_{n l}=\left(\mathbf{1}^{\otimes n}-R_{k}\right) p^{\oplus(l-1)} \oplus_{r} \oplus_{p}^{\oplus(n-l-1)} \equiv \\
p^{\oplus(k-1)} \bigoplus_{r} \oplus_{p}^{\oplus(l-k-2)} \oplus_{r} \oplus_{p}^{\oplus(n-l-1)} \equiv\left(\mathbf{1}^{\otimes n}-R_{l}\right) r_{n k} \quad\left(\bmod . j_{n-1}\right) .
\end{gathered}
$$

Thus $r_{n k}+R_{k} r_{n l} \equiv r_{n l}+R_{l} r_{n k} \quad\left(\bmod . j_{n-1}\right)$ and (3.35) follows. ad (ii). In virtue of (3.12)

$$
\begin{gathered}
r_{n k}+R_{k} r_{n, k+1}+R_{k} R_{k+1} r_{n k} \equiv \\
r_{n, k+1}+R_{k+1} r_{n k}+R_{k+1} R_{k} r_{n, k+1} \quad\left(\bmod . j_{n-1}\right)
\end{gathered}
$$

and (3.35) follows also in this case.

Thus in formula (3.32) we can use any minimal decomposition (3.31) for computations modulo $j_{n-1}$. We shall prove

$$
R_{k} r_{n} \equiv r_{n}-r_{n k} \quad\left(\bmod . j_{n-1}\right), \quad n=2,3, \ldots
$$


Let $\pi \in \Pi_{n}$ be such that $\pi^{-1}(k)<\pi^{-1}(k+1)$ and (3.31) be a minimal decomposition. Then $\pi^{\prime}=t_{k} \pi$ satisfies $\pi^{\prime-1}(k)>\pi^{\prime-1}(k+1)$ and has minimal decomposition $\pi^{\prime}=t_{k} t_{i_{1}} \cdot \ldots \cdot t_{i_{s}}$. In such a way we get all $\pi^{\prime}$ such that $\pi^{\prime-1}(k)>\pi^{\prime-1}(k+1)$, each one exactly once. Due to (3.32) and (3.35),

$$
r_{n \pi^{\prime}} \equiv R_{k} r_{n \pi}+r_{n k} \quad\left(\bmod . j_{n-1}\right),
$$

$R_{\pi^{\prime}}=R_{k} R_{\pi}$. Multiplying both sides by $R_{k}-\mathbf{1}^{\otimes n}$ and using

$$
R_{k} r_{n k}=-r_{n k}
$$

(it follows from $R r=-r$ ), we get

$$
\left(R_{k}-\mathbf{1}^{\otimes n}\right)\left(r_{n \pi}+r_{n \pi^{\prime}}\right) \equiv-2 r_{n k} \quad\left(\bmod . j_{n-1}\right),
$$

$\left(R_{k}-\mathbf{1}^{\otimes n}\right)\left(R_{\pi}+R_{\pi^{\prime}}\right)=0$. Thus

$$
\left(R_{k}-\mathbf{1}^{\otimes n}\right) r_{n}=\frac{1}{n !} \sum_{\pi}\left(R_{k}-\mathbf{1}^{\otimes n}\right)\left(r_{n \pi}+r_{n \pi^{\prime}}\right) \equiv-r_{n k} \quad\left(\bmod . j_{n-1}\right)
$$

and (3.36) is proved. Moreover,

$$
\left(R_{k}-\mathbf{1}^{\otimes n}\right) S_{n}=\frac{1}{n !} \sum_{\pi}\left(R_{k}-\mathbf{1}^{\otimes n}\right)\left(R_{\pi}+R_{\pi^{\prime}}\right)=0 .
$$

Thus $S_{n}^{2}=\frac{1}{n !} \sum_{\pi} R_{\pi} S_{n}=\frac{1}{n !} \sum_{\pi} S_{n}=S_{n}$. Using (3.36), (3.37) and mathematical induction w.r.t. the number of transpositions in a minimal decomposition of $\pi$, one gets $R_{\pi} r_{n} \equiv r_{n}-r_{n \pi}\left(\bmod . j_{n-1}\right)$. Therefore

$$
S_{n} r_{n} \equiv r_{n}-\frac{1}{n !} \sum_{\pi} r_{n \pi}=r_{n}-r_{n}=0 \quad\left(\bmod . j_{n-1}\right) .
$$

Using $S_{n} \tilde{p}^{\oplus n} \equiv \tilde{p}^{\oplus n}-r_{n}(\bmod . j)$ and (3.40), $\left(\mathbf{1}^{\otimes n}-S_{n}\right) \tilde{p}^{\oplus n} \equiv r_{n} \equiv\left(\mathbf{1}^{\otimes n}-\right.$ $\left.S_{n}\right) r_{n}$ (mod.j). Thus the elements (3.33) belong to $j$.

Let $\tilde{j}$ be the left module generated by (3.33). Then $\tilde{j} \subset j$. We shall prove by mathematical induction that $j_{n} \subset \tilde{j}$. It is true for $n=2$ since

$$
\left(\mathbf{1}^{\otimes 2}-R\right) \tilde{p}^{\oplus 2}-r=2\left(\mathbf{1}^{\otimes 2}-S_{2}\right)\left(\tilde{p}^{\oplus 2}-r_{2}\right)
$$

(we use $S_{2}=\frac{1}{2}+\frac{1}{2} R, r_{2}=\frac{1}{2} r, S_{2} r_{2}=0$ ). If it is true for $n-1$ then using (3.36) and (3.39), we get

$$
\left(\mathbf{1}^{\otimes n}-R_{k}\right) \tilde{p}^{\oplus n}-r_{n k} \equiv\left(\mathbf{1}^{\otimes n}-R_{k}\right)\left(\tilde{p}^{\oplus n}-r_{n}\right)=
$$




$$
\left(R_{k}-\mathbf{1}^{\otimes n}\right)\left(S_{n}-\mathbf{1}^{\otimes n}\right)\left(\tilde{p}^{\oplus n}-r_{n}\right) \equiv 0 \quad(\bmod . \tilde{j})
$$

and $j_{n} \subset \tilde{j}$. Therefore $j \subset \tilde{j}, j=\tilde{j}$.

We set $S_{0}=\operatorname{id}_{\mathbf{C}}$. Let $\alpha^{\prime}=\left\{\alpha^{\prime}{ }_{i n}: i=1, \ldots, \operatorname{dim} S_{n}\right\}$ be a basis of $\operatorname{im} S_{n}$, $\beta^{\prime}=\left\{\beta^{\prime}{ }_{j n}: j=1, \ldots, \operatorname{dim}\left(\mathbf{1}^{\otimes n}-S_{n}\right)\right\}$ be a basis of $\operatorname{im}\left(\mathbf{1}^{\otimes n}-S_{n}\right)$. Then $\alpha^{\prime} \sqcup \beta^{\prime}$ is a basis of $\left(\mathbf{C}^{|\mathcal{I}|}\right)^{\otimes n}$. We denote by $\alpha \sqcup \beta$ the dual basis. In particular,

$$
\alpha^{i n}\left(\mathbf{1}^{\otimes n}-S_{n}\right)=0 .
$$

We set $\mathcal{B}^{\prime}=\tilde{\mathcal{B}} / j$ and $p^{\prime}{ }_{k}=\lambda\left(\tilde{p}_{k}\right)$ where $\lambda: \tilde{\mathcal{B}} \longrightarrow \mathcal{B}^{\prime}$ is the canonical mapping.

Corollary 3.3 Let $K_{N}=\left\{\beta^{\text {in }}\left(\tilde{p}^{\oplus n}-r_{n}\right): i=1, \ldots, \operatorname{dim}\left(\mathbf{1}^{\otimes n}-S_{n}\right), n=\right.$ $2,3, \ldots, N\}, L_{N}^{\prime}=\left\{\alpha^{i n}\left(p^{\oplus n}\right): i=1, \ldots, \operatorname{dim} S_{n}, n=0,1,2, \ldots, N\right\}$. Then $K_{\infty}$ is a basis of $j, L_{\infty}^{\prime}$ is a basis of $\mathcal{B}^{\prime}, K_{N}$ is a basis of $j^{N}=j \cap \tilde{\mathcal{B}}^{N}$, $L^{\prime}{ }_{N}$ is a basis of

$$
\mathcal{B}^{\prime N}=\mathcal{A} \cdot \operatorname{span}\left\{p_{i_{1}}^{\prime} \cdot \ldots \cdot p_{i_{n}}^{\prime}: i_{1}, \ldots, i_{n}=1, \ldots,|\mathcal{I}|, n=0,1, \ldots, N\right\}
$$

(we treat $j, j^{N}, \mathcal{B}^{\prime}, \mathcal{B}^{\prime N}$ as the left modules).

Proof.

$$
\mathbf{1}^{\otimes n}-S_{n}=\sum_{i} \beta^{\prime}{ }_{i n} \beta^{i n}, \quad \beta^{j n}\left(\mathbf{1}^{\otimes n}-S_{n}\right)=\beta^{j n},
$$

hence $j$ is the left module generated by $K_{\infty}$. On the other hand, a finite combination $\sum a_{i n} \beta^{i n}\left(\tilde{p}^{\oplus n}-r_{n}\right), a_{i n} \in \mathcal{A}$, belongs to $\tilde{\mathcal{B}}^{N}$ iff $a_{i n}=0$ for $n>N$ (Lemma 1.1 and linear independence of $\beta^{i n}$ for given $n$ ). Therefore $j^{N}$ is generated by $K_{N}$ and (taking $N=0$ ) elements of $K_{\infty}$ are linearly independent over $\mathcal{A}$. Hence, $K_{\infty}$ is a basis of $j, K_{N}$ is a basis of $j^{N}$. Using $\mathbf{1}^{\otimes n}=\sum \alpha^{\prime}{ }_{i n} \alpha^{i n}+\sum \beta^{\prime}{ }_{i n} \beta^{i n}, K_{N} \sqcup\left\{\alpha^{i n}(\tilde{p})^{\oplus n}: i=1,2, \ldots, \operatorname{dim} S_{n}, n=\right.$ $0,1,2, \ldots, N\}$ is a basis of $\tilde{\mathcal{B}}^{N}, N \in \mathbf{N} \cup\{\infty\}\left(\tilde{\mathcal{B}}^{\infty}=\tilde{\mathcal{B}}\right)$. Thus $\mathcal{B}^{\prime}=$ $\tilde{\mathcal{B}}^{N} / j=\tilde{\mathcal{B}}^{N} / j^{N}$ has a basis $L^{\prime}{ }_{N}, N \in \mathbf{N} \cup\{\infty\}\left(\mathcal{B}^{\prime \infty}=\mathcal{B}^{\prime}\right)$.

Corollary 3.4 The left module $j^{N}=j \cap \tilde{\mathcal{B}}^{N}$ is generated by (3.33) for $n=$ $2,3, \ldots, N$. In particular, $j \cap \tilde{\mathcal{B}}^{1}=\{0\}, j \cap \tilde{\mathcal{B}}^{2}=j_{2}$.

Proof. It follows from Corollary 3.3, (3.43) and (3.41). 
Proposition 3.5 With the assumptions of Theorem 3.1, if $J$ satisfies the condition i) of Theorem 3.1 and $J \cap \tilde{\mathcal{B}}^{2}=j_{2}$ then $J=j$.

Proof. Clearly $j \subset J$. Let $J^{\prime}=J / j \subset \mathcal{B}^{\prime}$ and $N$ be the minimal number such that ${J^{\prime N}}^{N}=J^{\prime} \cap \mathcal{B}^{\prime N} \neq\{0\}$. Therefore $N \geq 3, \Delta J^{N} \subset J^{\prime N} \otimes \mathcal{A}+\mathcal{A} \otimes J^{\prime N}$. Let $0 \neq x \in J^{\prime N}$. Then

$$
x=\sum_{\substack{i=1, \ldots, \operatorname{dim} \\ n \leq N}} S_{n} a_{i n} \alpha^{i n} p^{\oplus_{n}} .
$$

We set $\mathcal{B}^{\prime}{ }_{n}=\mathcal{A} \cdot \operatorname{span}\left\{\alpha^{i n} p^{\Uparrow n}: i=1,2, \ldots, \operatorname{dim} S_{n}\right\}, \mathcal{B}^{\prime}=\oplus_{n=o}^{\infty} \mathcal{B}_{n}^{\prime}$. The component of $\Delta x$ belonging to $\mathcal{B}^{\prime}{ }_{N-1} \otimes \mathcal{B}^{\prime}{ }_{1}$ equals 0 . Thus

$$
\begin{gathered}
0 \equiv \sum_{i=1, \ldots, \mathrm{dim} S_{N}} \Delta\left(a_{i N}\right) \alpha_{j_{1} \ldots j_{N}}^{i N} \times \\
\sum_{k=1}^{N}{p^{\prime}}_{j_{1}}^{\prime} \cdot \ldots \cdot p_{j_{k-1}}^{\prime} \Lambda_{j_{k} m} p^{\prime}{ }_{j_{k+1}} \cdot \ldots \cdot p^{\prime}{ }_{j_{N}} \otimes p^{\prime}{ }_{m}\left(\bmod . \mathcal{B}^{\prime N-2} \otimes \mathcal{B}^{\prime}{ }_{1}\right) .
\end{gathered}
$$

In short,

$$
0 \equiv \sum_{i} \Delta\left(a_{i N}\right) \sum_{k=1}^{N} \alpha^{i N}\left[\left(p^{\curvearrowright(k-1)} \oplus \Lambda \oplus p^{\curvearrowright(N-k)}\right) \oplus p^{\prime}\right] .
$$

Since (1.6) holds in $\tilde{\mathcal{B}}, p^{\prime} \oplus \Lambda \equiv R\left(\Lambda \oplus p^{\prime}\right)(\bmod . \mathcal{A})$. Using (3.42), $\alpha^{i N}=$ $\alpha^{i N} S_{N}=\alpha^{i N} S_{N} R_{k}=\alpha^{i N} R_{k}$ and all components in the second sum are equal modulo $\mathcal{B}^{\prime N-2} \otimes \mathcal{B}^{\prime}$. We get

$$
\begin{gathered}
0 \equiv \sum_{i} \Delta\left(a_{i N}\right) \alpha^{i N}\left[\left(p^{\prime} \oplus \ldots \oplus p^{\prime} \oplus \Lambda\right) \otimes p^{\prime}\right] \\
0 \equiv \sum_{i} a_{i N}^{(1)} \alpha_{j_{1} \ldots j_{N}}^{i N} p_{j_{1}}^{\prime} \ldots p_{j_{N-1}}^{\prime} \Lambda_{j_{N} m} \otimes a_{i N}^{(2)} \quad\left(\bmod .{\mathcal{B}^{\prime}}^{N-2} \otimes \mathcal{A}\right) .
\end{gathered}
$$

Acting by id $\otimes \epsilon$ and multiplying by $\Lambda_{m k}^{-1} p_{k}^{\prime}$, one has

$$
0 \equiv \sum_{i} a_{i N} \alpha^{i N} p^{{ }^{\oplus N}} \quad\left(\bmod . \mathcal{B}^{N-1}\right) .
$$


Using Corollary 3.3 , one obtains $a_{i N}=0, i=1, \ldots, \operatorname{dim} S_{N}, x \in J^{\prime} \cap \mathcal{B}^{\prime N-1}=$ $\{0\}$, contradiction. We get $J^{\prime}=\{0\}, J=j$.

End of proof of Theorem 3.1. We use the above facts.

Corollary 3.6 Let $L_{N}=\left\{\alpha^{i n} p^{\oplus n}: i=1,2, \ldots, \operatorname{dim} S_{n}, n=0,1,2, \ldots, N\right\}$. Then $L_{\infty}$ is a basis of $\mathcal{B}, L_{N}$ is a basis of $\mathcal{B}^{N}=\mathcal{A} \cdot \operatorname{span}\left\{p_{i_{1}} \ldots p_{i_{n}}\right.$ : $\left.i_{1}, \ldots, i_{n} \in \mathcal{I}, n=0,1, \ldots, N\right\}$ (we treat $\mathcal{B}, \mathcal{B}^{N}$ as left modules). In particular,

$$
\operatorname{dim}_{\mathcal{A}} \mathcal{B}^{N}=\sum_{n=0}^{N} \operatorname{dim} S_{n} .
$$

Remark 3.7 Assume that the condition c. doesn't hold. Then we introduce $\eta_{i} \in \mathcal{A}^{\prime}$ as before, so (2.3) holds. But on the right hand side of (2.5) (for $a=v_{A C}, b=w_{B D}, v, w \in \operatorname{Irr} H$ ) we must add $L_{m A B, C D}^{v w}$ where $L^{v w} \in \operatorname{Mor}(v \oplus w, \Lambda \oplus v(\mathrm{D})$ and we don't have (2.6), (2.18). Nevertheless, (1.8) is valid. Therefore we get admissible $\eta_{i}$ in the following way. Let matrix elements of nontrivial irreducible representations $\left\{w^{m}\right\}_{m \in M}$ generate $\mathcal{A}$ as algebra with $I$. We put $\eta_{i}(I)=0$, assume some values of $\eta_{i}\left(w_{A B}^{m}\right)$ and using (2.3) compute $\phi_{i}\left(w_{A B}^{m}\right)$. Then we have the condition that $\rho$ of (1.8) preserves all the relations among $w_{A B}^{m}$. We choose $\eta_{i}\left(\Lambda_{j s}\right)$ so that (2.11) is satisfied ( $\eta_{i}$ are in general not determined uniquely by $\left.\phi_{i}\right)$. We also have an additional condition that (2.19) holds for $b$ being matrix elements of $\left\{w^{m}\right\}_{m \in M}$ (due to Remark 2.0 it implies (2.19) for all $b \in \mathcal{A}$ and using it we get $A_{\alpha r}=0$ and bimodule condition for $j_{2}$ in Theorem 2.3). Proposition 3.14 holds provided $\operatorname{Mor}(1, \Lambda)=\{0\}, \operatorname{Mor}(\Lambda, \Lambda \oplus \Lambda)=\{0\}$ (otherwise we replace $\operatorname{Mor}(\mathcal{P} \oplus \mathcal{P}, \mathcal{P} \oplus \mathcal{P})$ by $V_{0}$ in both places where $V_{0}$ is some linear subspace of $\operatorname{Mor}(\mathcal{P} \oplus \mathcal{P}, \mathcal{P} \oplus \mathcal{P}))$. Moreover, in Proposition 3.14.2.c there is $B[Z \otimes \mathbf{1}+(R \otimes \mathbf{1})(\mathbf{1} \otimes Z)] m$ on the right hand side of (3.59) and also one more condition (3.61). We don't get Proposition 4.5.2, Proposition 4.8 and Corollary 4.9. In Proposition 4.5.3, Proposition 5.5, Theorem 5.6 and Proposition 5.7 we assume that $\Lambda$ is a nontrivial representation. With such corrections, the results of Sections $2-5$ are still valid. 
Corollary 3.8a) $\mathcal{B}$ is the universal unital algebra generated by $\mathcal{A}$ and $p_{i}$ $(i \in \mathcal{I})$ with the relations $I_{\mathcal{B}}=I_{\mathcal{A}}$,

$$
\begin{array}{r}
p_{s} a=\left(a * f_{s t}\right) p_{t}+a * \eta_{s}-\Lambda_{s t}\left(\eta_{t} * a\right), \quad a \in \mathcal{A}, \\
\left(R-\mathbf{1}^{\otimes 2}\right)_{k l, i j}\left(p_{i} p_{j}-\eta_{i}\left(\Lambda_{j s}\right) p_{s}+T_{i j}-\Lambda_{i m} \Lambda_{j n} T_{m n}\right)=0 .
\end{array}
$$

b) $\mathcal{B}$ is the universal unital algebra generated by $\mathcal{A}$ and $p_{1}, \ldots, p_{s}$ with the relations $I_{\mathcal{B}}=I_{\mathcal{A}}$,

$$
\begin{gathered}
(\mathcal{P} \oplus w) N_{w}=N_{w}(w \oplus \mathcal{P}), \quad w \in \operatorname{Rep} H, \\
R_{P}(\mathcal{P} \oplus \mathcal{P})=(\mathcal{P} \bigcirc \mathcal{P}) R_{P},
\end{gathered}
$$

where $\mathcal{P}$ is given by the condition 3.,

$$
\begin{gathered}
R_{P}=\left(\begin{array}{cccc}
R & Z & -R \cdot Z & \left(R-\mathbf{1}^{\otimes 2}\right) T \\
0 & 0 & \mathbf{1} & 0 \\
0 & \mathbf{1} & 0 & 0 \\
0 & 0 & 0 & 1
\end{array}\right), N_{w}=\left(\begin{array}{cc}
G_{w} & H_{w} \\
0 & \mathbf{1}_{w}
\end{array}\right), \\
\left(G_{w}\right)_{i C, D j}=f_{i j}\left(w_{C D}\right),\left(H_{w}\right)_{i C, D}=\eta_{i}\left(w_{C D}\right), R=G_{\Lambda}, Z=H_{\Lambda} .
\end{gathered}
$$

Remark 3.9 For $\eta=0, T=0$ (that choice always satisfies the conditions (2.14), (3.1), (3.9)) we get $p_{s} \Lambda_{i j}=R_{s i, m t} \Lambda_{m j} p_{t}, R_{k l, i j} p_{i} p_{j}=p_{k} p_{l}$ (cf [13], [8]).

Remark 3.10 In (3.45) it suffices to take a being generators of $\mathcal{A}$ (as algebra with unity), in (3.47) it suffices to take $\left\{w^{m}\right\}_{m \in M} \subset$ Rep $H$ such that matrix elements of $w^{m}$ generate $\mathcal{A}$.

Remark 3.11 Replacing $T$ by $T^{\prime}=\frac{1}{2}\left(\mathbf{1}^{\otimes 2}-R\right) T$, we don't change (3.46). One has $R T^{\prime}=-T^{\prime}$. So in the following we can (and will) assume

$$
R T=-T .
$$


Proof. a) follows from Theorem 3.1. Due to (2.3) and (1.7) it suffices to take $a$ as generators.

ad b) Let $a=w_{m n}, m, n=1, \ldots, \operatorname{dim} w$. Then (1.5) implies

$$
(\Lambda \oplus w) G_{w}=G_{w}(w \oplus \Lambda)
$$

(3.45) is equivalent to

$$
p \oplus w=G_{w}(w \oplus p)+H_{w} w-(\Lambda \oplus w) H_{w} .
$$

We can rewrite these two equations as (3.47). One can replace (3.46) by

$$
\left(R-\mathbf{1}^{\otimes 2}\right)(p \oplus p-Z \cdot p+T-(\Lambda \oplus \Lambda) T)=0 .
$$

Using (3.51) for $w=\Lambda$, this is equivalent to (3.48).

Proposition $3.12 \mathcal{B}$ is a Hopf algebra (with invertible coinverse).

Proof. Let $w, w^{\prime} \in \operatorname{Irr} H$. Then

$$
w \oplus w^{\prime} \simeq \oplus_{w^{\prime \prime} \in \operatorname{Irr} H} c_{w w^{\prime}}^{w^{\prime \prime}} w^{\prime \prime}
$$

for some $c_{w w^{\prime}}^{w^{\prime \prime}} \in \mathbf{N}$. Thus there exist linearly independent $S_{w w^{\prime} w^{\prime \prime}}^{\alpha} \in \operatorname{Mor}\left(w^{\prime \prime}, w \oplus w^{\prime}\right)$, $\alpha=1, \ldots, c_{w w^{\prime}}^{w^{\prime \prime}}$. Then $\mathcal{A}$ is the algebra generated by matrix elements of unequivalent irreducible representations of $H$ satisfying

$$
\left(w \bigcirc w^{\prime}\right) S_{w w^{\prime} w^{\prime \prime}}^{\alpha}=S_{w w^{\prime} w^{\prime \prime}}^{\alpha} w^{\prime \prime}, \quad w, w^{\prime}, w^{\prime \prime} \in \operatorname{Irr} H, \quad \alpha=1, \ldots, c_{w w^{\prime}}^{w^{\prime \prime}}
$$

We conclude that $\mathcal{B}$ is the universal algebra generated by (matrix elements of) a set of representations of $G(\mathcal{P}$ and $w \in \operatorname{Irr} H)$ satisfying (3.47), (3.48), (3.53),

$$
\mathcal{P}=I \oplus \mathcal{P}, \quad \mathcal{P} i=i \Lambda \text { and } s \mathcal{P}=I s
$$

where $i: \mathbf{C}^{|\mathcal{I}|} \longrightarrow \mathbf{C}^{|\mathcal{I}|} \oplus \mathbf{C}, \quad s: \mathbf{C}^{|\mathcal{I}|} \oplus \mathbf{C} \longrightarrow \mathbf{C}$ are the canonical mappings, $I$ is the trivial representation of $H$. Thus the relations are given by morphisms. Moreover, these representations are invertible:

$$
\mathcal{P}^{-1}=\left(\begin{array}{cc}
\Lambda^{-1} & -\Lambda^{-1} p \\
0 & I
\end{array}\right)
$$


$w^{-1}=S(w)$ for $w \in \operatorname{Irr} H$. Using the arguments of 110 or $\| 16$, we get that $\mathcal{B}$ has a coinverse $S$. Similarly, $\mathcal{P}^{T}$ and $w^{T}, w \in \operatorname{Irr} H$, are invertible representations of $G^{\mathrm{opp}}$, where Poly $\left(G^{\mathrm{opp}}\right)=(\mathcal{B}, \tau \Delta)$ (coinverse of $\mathcal{A}$ is invertible). Hence $(\mathcal{B}, \tau \Delta)$ has a coinverse $S^{\prime}$, by the general theory $S^{\prime}=S^{-1}$.

Let

$$
\tau^{k l}=\left(R-\mathbf{1}^{\otimes 2}\right)_{k l, i j} \tau_{i j},
$$

where $\tau_{i j}$ are defined in Theorem 2.3.

\section{Proposition 3.13}

1) 2.14) is equivalent to

$$
(\Lambda \oplus \Lambda)_{k l, i j}\left(\tau^{i j} * b\right)=b * \tau^{k l}, \quad b \in S
$$

where $S$ is a set generating $\mathcal{A}$ as an algebra with unity

2)

$$
\tau^{i j}(a b)=\left(f_{j s} * f_{i m}\right)(a) \tau^{m s}(b)+\tau^{i j}(a) \epsilon(b) \quad(a, b \in \mathcal{A}), \quad \tau^{i j}(I)=0 .
$$

Proof. 1) follows from Remark 2.6, (1.15), (3.5) and (1.12). 2) follows from Proposition 2.4, (1.16), (3.5) and

$$
R_{k l, i j}\left(f_{j s} * f_{i m}\right)=\left(f_{l j} * f_{k i}\right) R_{i j, m s}
$$

( we get it acting $f_{i j}$ on (1.5)).

Proposition 3.14 Let $R \notin \mathbf{C} \mathbf{1}^{\otimes 2}$. One has

1) $\operatorname{Mor}(\mathcal{P} \oplus \mathcal{P}, \mathcal{P} \oplus \mathcal{P})=\mathbf{C} \cdot \mathrm{id} \oplus \mathbf{C} R_{P} \oplus\left\{m_{P}:(\Lambda \oplus \Lambda) m=m\right\}$ where $R_{P}$ is given by (3.49) and

$$
m_{P}=\left(\begin{array}{cccc}
0 & 0 & 0 & m \\
0 & 0 & 0 & 0 \\
0 & 0 & 0 & 0 \\
0 & 0 & 0 & 0
\end{array}\right)
$$


2) $W \in \operatorname{Mor}(\mathcal{P} \oplus \mathcal{P}, \mathcal{P} \oplus \mathcal{P})$ satisfies

$$
(W \otimes \mathbf{1})(\mathbf{1} \otimes W)(W \otimes \mathbf{1})=(\mathbf{1} \otimes W)(W \otimes \mathbf{1})(\mathbf{1} \otimes W)
$$

if and only if

a) $W=x \cdot \operatorname{id}(x \in \mathbf{C} \backslash\{0\})$ or

b) $W \in\left\{m_{P}:(\Lambda \oplus \Lambda) m=m\right\}$ or

c) $W=y \cdot\left(R_{P}+m_{P}\right)$ for $y \in \mathbf{C} \backslash\{0\}$ and $m$ such that $(\Lambda \oplus \Lambda) m=m$ provided that

$$
\begin{gathered}
{\left[\left(R-\mathbf{1}^{\otimes 2}\right) \otimes \mathbf{1}\right] F=0,} \\
A_{3}(Z \otimes \mathbf{1}-\mathbf{1} \otimes Z) T=0 .
\end{gathered}
$$

Those $W$ are invertible if and only if we have the case a) or c).

Remark 3.15 Examples of $R$-matrices for inhomogeneous quantum groups were given e.g. in [8], [13], [3], 财].

Proof. ad 1) One has

$$
\mathcal{P} \oplus \mathcal{P}=\left(\begin{array}{cccc}
\Lambda \oplus \Lambda & \Lambda \oplus p & p \oplus \Lambda & p \oplus p \\
0 & \Lambda & 0 & p \\
0 & 0 & \Lambda & p \\
0 & 0 & 0 & I
\end{array}\right)
$$

We assume

$$
W=\left(\begin{array}{cccc}
A & B & E & F \\
C & D & G & H \\
J & K & N & P \\
L & M & Q & U
\end{array}\right) \in \operatorname{Mor}(\mathcal{P} \oplus \mathcal{P}, \mathcal{P} \oplus \mathcal{P})
$$

It gives a set of linear relations on matrices $A, B, \ldots, U$. Using (3.52) and

$$
p \oplus \Lambda=R(\Lambda \oplus p)+Z \Lambda-(\Lambda \oplus \Lambda) Z
$$

(it is (3.51) for $w=\Lambda$ ) one can solve them and get $A=b+a R, B=a Z$, $E=-a R Z, F=a\left(R-\mathbf{1}^{\otimes 2}\right) T+m, D=N=b \mathbf{1}, G=K=a \mathbf{1}, U=a+b$, 
$C=H=J=P=L=M=Q=0$, where $a, b, k \in \mathbf{C},(\Lambda \bigcirc \Lambda) m=m$. It means $W=b \cdot \mathrm{id}+a \cdot R_{P}+m_{P}$ and 1) follows.

ad 2) We set $l=Z \otimes \mathbf{1}+(R \otimes \mathbf{1})(\mathbf{1} \otimes Z)$. Using (2.5), we get $l_{i j k, r s}=$ $\eta_{i}\left((\Lambda \bigcirc \Lambda)_{j k, r s}\right)$ and $($ see $(\underline{1.12}))$

$$
(\mathbf{1} \otimes R) l=l R .
$$

Moreover, $(\Lambda \oplus \Lambda) m=m$ gives

$$
l m=0
$$

and (acting by $f_{i j}$ )

$$
(R \otimes \mathbf{1})(\mathbf{1} \otimes R)(m \otimes \mathbf{1})=\mathbf{1} \otimes m,
$$

hence (using $R^{2}=\mathbf{1}^{\otimes 2}$ )

$(\mathbf{1} \otimes R)(R \otimes \mathbf{1})(\mathbf{1} \otimes m)=m \otimes \mathbf{1}$.

We shall check for which $m$ and $x, y \in \mathbf{C}, W=x \cdot \mathrm{id}+y \cdot R_{P}+m_{P}$ satisfies (3.57). Since $\mathcal{P}$ acts in $\mathbf{C}^{|\mathcal{I}|} \oplus \mathbf{C}, W$ acts on

$$
\mathbf{C}^{|\mathcal{I}|} \otimes \mathbf{C}^{|\mathcal{I}|} \oplus \mathbf{C}^{|\mathcal{I}|} \otimes \mathbf{C} \oplus \mathbf{C} \otimes \mathbf{C}^{|\mathcal{I}|} \oplus \mathbf{C} \otimes \mathbf{C} .
$$

Denoting the standard basis elements in $\mathbf{C}^{|\mathcal{I}|} \oplus \mathbf{C}$ by $e_{i}(i \in \mathcal{I})$ and $f$, one gets

$$
\begin{aligned}
R_{P}\left(e_{i} \otimes e_{j}\right) & =R_{k l, i j} e_{k} \otimes e_{l}, \\
R_{P}\left(e_{i} \otimes f\right) & =Z_{k l, i} e_{k} \otimes e_{l}+f \otimes e_{i}, \\
R_{P}\left(f \otimes e_{i}\right) & =-(R Z)_{k l, i} e_{k} \otimes e_{l}+e_{i} \otimes f, \\
R_{P}(f \otimes f) & =\left(\left(R-\mathbf{1}^{\otimes 2}\right) T\right)_{i j} e_{i} \otimes e_{j}+f \otimes f, \\
m_{P}\left(e_{i} \otimes e_{j}\right) & =0, \\
m_{P}\left(e_{i} \otimes f\right) & =0, \\
m_{P}\left(f \otimes e_{i}\right) & =0, \\
m_{P}(f \otimes f) & =m_{i j} e_{i} \otimes e_{j} .
\end{aligned}
$$


Let us restrict ourselves to $\mathbf{C}^{|\mathcal{I}|} \otimes \mathbf{C}^{|\mathcal{I}|} \otimes \mathbf{C}^{|\mathcal{I}|}$. Then (3.57) gives an analogous formula for $x \cdot \mathbf{1}^{\otimes 2}+y \cdot R$. Using (3.11) and $R^{2}=\mathbf{1}^{\otimes 2}$, it means $x^{2} y(R \otimes \mathbf{1}-\mathbf{1} \otimes R)=0, x=0$ or $y=0(R \otimes \mathbf{1}=\mathbf{1} \otimes R$ would mean $V \otimes \mathbf{C}^{4}=\mathbf{C}^{4} \otimes V$ where $V=\operatorname{ker}\left(R+\mathbf{1}^{\otimes 2}\right), R \in \mathbf{C 1}^{\otimes 2}$, contradiction).

Setting $x \neq 0, y=0$ and applying both sides of (3.57) to $f \otimes f \otimes e_{k}$, one obtains $m_{i j}\left(e_{i} \otimes e_{j} \otimes e_{k}\right)=0, m=0$. Clearly $x \neq 0, y=0, m=0$ gives a solution of (3.57). The same holds for $x=y=0$ (both sides of (3.57) equal $0)$. It remains to consider $W=y\left(R_{P}+m_{P}\right)$ for $y \neq 0$. In order to check (3.57) we may assume $y=1$. Using (3.65), we find that (3.57) on $e_{i} \otimes e_{j} \otimes e_{k}$ follows from (3.11), on $e_{i} \otimes e_{j} \otimes f, e_{i} \otimes f \otimes e_{j}, f \otimes e_{i} \otimes e_{j}$ is equivalent to (3.61), on $e_{i} \otimes f \otimes f, f \otimes e_{i} \otimes f, f \otimes f \otimes e_{i}$ is equivalent to (3.58) (we use (3.30), (3.61) and (3.64) ), on $f \otimes f \otimes f$ is equivalent to $B l s=0$ where $B$ is given by (3.14), $s=s_{0}+m, s_{0}=\left(R-\mathbf{1}^{\otimes 2}\right) T=-2 T$ (see (3.50)). Using (3.62) and $\left[\mathbf{1} \otimes\left(\mathbf{1}^{\otimes 2}+R\right)\right] l s_{0}=0$ (which follows from (3.61)), we get

$$
B l s=B l s_{0}=\frac{1}{2} A_{3} l s_{0}=-A_{3}(Z \otimes \mathbf{1}-\mathbf{1} \otimes Z) T
$$

and $B l s=0$ is equivalent to ( $\overline{3.59}$ ).

Invertibility condition is obvious (in the case c) we use the existence of $\left.R_{P}^{-1}=R_{P}\right)$.

Remark 3.16 One can also consider the case when $\left(R+\mathbf{1}^{\otimes 2}\right)\left(R-Q \mathbf{1}^{\otimes 2}\right)=0$ where $Q \neq 0, \pm 1$ is not a root of unity. Then $(\rho+\mathrm{id})(\rho-Q \mathrm{id})=0$ and we should replace everywhere $R-\mathbf{1}^{\otimes 2}$ by $R-Q \mathbf{1}^{\otimes 2}, R_{k l, i j} p_{i} p_{j}=p_{k} p_{l}$ by $R_{k l, i j} p_{i} p_{j}=Q p_{k} p_{l}$,

$$
\begin{aligned}
& A_{3}=Q^{3} \mathbf{1} \otimes \mathbf{1} \otimes \mathbf{1}-Q^{2} R \otimes \mathbf{1}-Q^{2} \mathbf{1} \otimes R+Q(R \otimes \mathbf{1})(\mathbf{1} \otimes R)+ \\
& Q(\mathbf{1} \otimes R)(R \otimes \mathbf{1})-(R \otimes \mathbf{1})(\mathbf{1} \otimes R)(R \otimes \mathbf{1}), \\
& A=(R \otimes \mathbf{1})(\mathbf{1} \otimes R)-Q(\mathbf{1} \otimes R)+Q^{2} \mathbf{1} \otimes \mathbf{1} \otimes \mathbf{1}, \\
& B=(\mathbf{1} \otimes R)(R \otimes \mathbf{1})-Q R \otimes \mathbf{1}+Q^{2} \mathbf{1} \otimes \mathbf{1} \otimes \mathbf{1}, \\
& R_{P}=\left(\begin{array}{cccc}
R & Z & (Q-1-R) \cdot Z & \left(R-Q \mathbf{1}^{\otimes 2}\right) T \\
0 & 0 & Q \mathbf{1} & 0 \\
0 & \mathbf{1} & (Q-1) \mathbf{1} & 0 \\
0 & 0 & 0 & Q
\end{array}\right)
\end{aligned}
$$


$n$ ! is replaced by

$$
(n)_{Q} !=\sum_{\pi \in \Pi_{n}} Q^{s(\pi)}=(1)_{Q}(2)_{Q} \cdot \ldots \cdot(n)_{Q}
$$

where $s(\pi)$ is the number of transpositions in the minimal decomposition of $\pi$ and $(k)_{Q}=1+Q+\ldots+Q^{k-1}$ (what concerns $S_{n}$ and $A_{3}$ see [5]),

$$
r_{n \pi}=Q^{s-1} r_{n i_{1}}+Q^{s-2} R_{i_{1}} r_{n i_{2}}+\ldots+R_{i_{1}} \cdot \ldots \cdot R_{i_{s-1}} r_{n i_{s}}
$$

where $s=s(\pi)$, in Remark 3.11 $T^{\prime}=\frac{1}{1+Q}\left(Q \mathbf{1}^{\otimes 2}-R\right) T$. In Proposition 3.14.2.c one has $W=y\left(R_{P}+m_{P}\right)^{ \pm 1},(\mathbf{1} \otimes R)(R \otimes \mathbf{1})(\mathbf{1} \otimes m)-m \otimes \mathbf{1}$ on the right hand side of (3.58) and additional condition $R m=-m$. In (1.4) one has $\frac{1}{(1+Q) c^{2}}\left(Q \mathbf{1}^{\otimes 2}-\hat{R}\right)$ instead of $\frac{1}{2 c^{2}}\left(\mathbf{1}^{\otimes 2}-\hat{R}\right)$. In $(4.14)$ one obtains $R_{a b, j l}^{-1}$ on the left hand side. In Proposition 4.5.2, Proposition 5.5, Theorem 5.6 and Proposition 5.7 we assume $|Q|=1$ (otherwise existence of the considered *-structure in $\mathcal{B}$ would imply $\left.R=-\mathbf{1}^{\otimes 2}, \mathcal{B}=\mathcal{A} \cdot \operatorname{span}\left\{I, p_{i}\right\}\right)$. With these corrections, all the results (in particular Remark 3.7) remain true but we do not get Proposition 4.8, Corollary 4.9 and there are small modifications in the proofs.

\section{Isomorphisms and $*$ structure}

In this Section we consider isomorphisms among inhomogeneous quantum groups as well as $*$-structures on them. Throughout the Section we assume that $\operatorname{Poly}(H)=(\mathcal{A}, \Delta)$ is a Hopf algebra satisfying the conditions a. $-\mathrm{c}$. and $\operatorname{Poly}(G)=(\mathcal{B}, \Delta)$ is the corresponding Hopf algebra as in Theorems 2.1 and 3.1. Then $G$ is called an inhomogeneous quantum group.

Proposition 4.1 Let $w \in \operatorname{Irr} G$. Then $w \in \operatorname{Irr} H$.

Proof. Let $W=\operatorname{span}\left\{w_{m n}: m, n=1, \ldots, \operatorname{dim} w\right\}$ and $s$ be the smallest natural number such that $W \subset \mathcal{B}^{s}\left(\mathcal{B}=\cup_{s} \mathcal{B}^{s}\right)$. Assume that $s>0$. Then $\Delta \mathcal{B}^{s} \subset \mathcal{B}^{s-1} \otimes \mathcal{B}^{s}+\mathcal{B}^{s} \otimes \mathcal{B}^{s-1}$. There exists $\phi \in\left(\mathcal{B}^{s}\right)^{\prime}$ such that $\phi_{\left.\right|_{\mathcal{B}^{s-1}}}=0$ and $\phi_{\left.\right|_{W}} \neq 0$. Therefore $(\mathrm{id} \otimes \phi) \Delta W \subset \mathcal{B}^{s-1}, w \phi(w) \in M_{\operatorname{dim} w}\left(\mathcal{B}^{s-1}\right)$. Moreover, one can choose $x \in \mathbf{C}^{\operatorname{dim} w}$ such that $\phi(w) x \neq 0$. We take $\phi(w) x$ as the first vector of a basis in the carrier vector space of $w$. Thus $w_{k 1} \in \mathcal{B}^{s-1}$, 
$k=1, \ldots, \operatorname{dim} w, w_{k l} \otimes w_{l 1}=\Delta w_{k 1} \in \mathcal{B}^{s-1} \otimes \mathcal{B}^{s-1}$. Using linear independence of $w_{l 1}(w \in \operatorname{Irr} G)$, we get $w_{k l} \in \mathcal{B}^{s-1}, W \subset \mathcal{B}^{s-1}$, contradiction. Thus $s=0, W \subset \mathcal{B}^{0}=\mathcal{A}$.

\section{Corollary 4.2}

$$
\mathcal{A}=\operatorname{span}\left\{w_{k l}: k, l=1, \ldots, \operatorname{dim} w, w \in \operatorname{Irr} G\right\} .
$$

Thus $\mathcal{B}$ determines $\mathcal{A}$ uniquely.

Proposition 4.3 Let $x \in \mathcal{B}, \Delta x \in \mathcal{A} \otimes \mathcal{B}+\mathcal{B} \otimes \mathcal{A}$. Then $x \in \mathcal{B}^{1}$.

Proof. Let $N$ be the minimal number such that $x \in \mathcal{B}^{N}$. Assume $N \geq 2$. Then

$$
x=\sum_{\substack{i=1, \ldots, \operatorname{dim} \\ n \leq N}} S_{n} a_{i n} \alpha^{i n} p^{\oplus n} .
$$

Using the same arguments as in the proof of Proposition 3.5, one gets $a_{i N}=0$, $i=1, \ldots, \operatorname{dim} S_{N}, x \in \mathcal{B}^{N-1}$, contradiction. Thus $x \in \mathcal{B}^{1}$.

\section{Proposition 4.4 One has}

1) Suppose that $\mathcal{B}, \mathcal{A}, \Delta, \Lambda, p, f, \eta, T$ and $\hat{\mathcal{B}}, \hat{\mathcal{A}}, \hat{\Delta}, \hat{\Lambda}, \hat{p}, \hat{f}, \hat{\eta}, \hat{T}$ describe two inhomogeneous quantum groups $G, \hat{G}$ and $\phi: \mathcal{B} \longrightarrow \hat{\mathcal{B}}$ be an isomorphism of bialgebras. Then $\phi(\mathcal{A})=\hat{\mathcal{A}}, \phi\left(\mathcal{B}^{1}\right)=\hat{\mathcal{B}}^{1}$. We denote $\phi_{\mathcal{A}}=\phi_{\left.\right|_{\mathcal{A}}}: \mathcal{A} \longrightarrow \hat{\mathcal{A}}$.

2) Moreover, let $\phi(\Lambda)=M \hat{\Lambda} M^{-1}$ for an invertible matrix $M$. Then

$$
\phi(p)=M(c \hat{p}+h-\hat{\Lambda} h),
$$

for some $c \in \mathbf{C} \backslash\{0\}, h_{s} \in \mathbf{C}(s \in \mathcal{I})$ and we can choose

$$
\begin{gathered}
\hat{f}=\left(M^{-1} f M\right) \circ \phi_{\mathcal{A}}^{-1} \\
\hat{\eta}=\frac{1}{c} M^{-1}(\eta+f M h-\epsilon M h) \circ \phi_{\mathcal{A}}^{-1}, \\
\hat{T}=\frac{1}{c^{2}}\left(M^{-1} \otimes M^{-1}\right) T+\frac{1}{2 c^{2}}\left(\mathbf{1}^{\otimes 2}-\hat{R}\right)\left[-\left(M^{-1} \otimes M^{-1}\right) Z M h+h \otimes h\right]
\end{gathered}
$$

where $Z=\eta(\Lambda)$,

$$
\hat{R}=\left(M^{-1} \otimes M^{-1}\right) R(M \otimes M)
$$


3) Let $\mathcal{B}, \mathcal{A}, \Delta, \Lambda, p, f, \eta, T$ describe an inhomogeneous quantum group, $\hat{\mathcal{A}}, \hat{\Delta}, \hat{\Lambda}$ satisfy the conditions a. - c., $\phi_{\mathcal{A}}: \mathcal{A} \longrightarrow \hat{\mathcal{A}}$ be an isomorphism of bialgebras such that $\phi_{\mathcal{A}}(\Lambda)=M \hat{\Lambda} M^{-1}$ for an invertible matrix $M$ and $c \in \mathbf{C} \backslash\{0\}$, $h_{s} \in \mathbf{C}(s \in \mathcal{I})$. Then there exists an inhomogeneous quantum group described by $\hat{\mathcal{B}}, \hat{\mathcal{A}}, \hat{\Delta}, \hat{\Lambda}, \hat{p}, \hat{f}, \hat{\eta}, \hat{T}$ and isomorphism of bialgebras $\phi: \mathcal{B} \longrightarrow \hat{\mathcal{B}}$ such that $\phi_{\mathcal{A}}=\phi_{\left.\right|_{\mathcal{A}}}$ and $(4.1)-(4.4)$ hold.

Proof. ad 1) According to Corollary 4.2,

$$
\begin{gathered}
\phi(\mathcal{A})=\operatorname{span}\left\{\phi\left(w_{k l}\right): k, l=1, \ldots, \operatorname{dim} w, w \in \operatorname{Irr} G\right\}= \\
\quad \operatorname{span}\left\{v_{k l}: k, l=1, \ldots, \operatorname{dim} v, v \in \operatorname{Irr} \hat{G}\right\}=\hat{\mathcal{A}} .
\end{gathered}
$$

Let $x \in \mathcal{B}^{1}$. Then $\Delta x \in \mathcal{B} \otimes \mathcal{A}+\mathcal{A} \otimes \mathcal{B}, \Delta \phi(x)=(\phi \otimes \phi) \Delta x \in \hat{\mathcal{B}} \otimes \hat{\mathcal{A}}+\hat{\mathcal{A}} \otimes \hat{\mathcal{B}}$. Using Proposition 4.3, we get $\phi(x) \in \hat{\mathcal{B}}^{1}$. Thus $\phi\left(\mathcal{B}^{1}\right) \subset \hat{\mathcal{B}}^{1}$. Interchanging $\mathcal{B}$ with $\hat{\mathcal{B}}$, one gets $\phi\left(\mathcal{B}^{1}\right)=\hat{\mathcal{B}}^{1}$.

ad 2) $\phi(p)=k \cdot \hat{p}+l$ for some $k_{i j}, l_{i} \in \hat{\mathcal{A}}$. Therefore

$$
\begin{gathered}
(k \hat{p}+l) \oplus I+M \hat{\Lambda} M^{-1} \oplus(k \hat{p}+l)=(\phi \otimes \phi)(p \oplus I+\Lambda \oplus p)= \\
(\phi \otimes \phi) \Delta p=\hat{\Delta} \phi(p)=\hat{\Delta}(k)(\hat{p} \oplus I+\hat{\Lambda} \oplus \hat{p})+\hat{\Delta}(l) .
\end{gathered}
$$

We get $\hat{\Delta}(k)=k \oplus I, k_{i j} \in \mathbf{C}, M \hat{\Lambda} M^{-1} k=k \hat{\Lambda}, l \oplus I+M \hat{\Lambda} M^{-1} \oplus l=\hat{\Delta}(l)$. Thus $k=c \cdot M$ and (cf (2.10) and later formulae) $l=\hat{h}-M \hat{\Lambda} M^{-1} \hat{h}$ for some $c \in \mathbf{C} \backslash\{0\}, \hat{h}_{s} \in \mathbf{C}(s \in \mathcal{I})$. Setting $h=M^{-1} \hat{h}$ one obtains (4.1).

Acting $\phi$ on the relation $p a=(a * f) p+a * \eta-\Lambda(\eta * a)$, we get

$$
\left.\begin{array}{l}
M(c \hat{p}+h-\hat{\Lambda} h) b= \\
\left(b * f \circ \phi_{\mathcal{A}}^{-1}\right) M(c \hat{p}+h-\hat{\Lambda} h)+ \\
b * \eta \circ \phi_{\mathcal{A}}^{-1}-M \hat{\Lambda} M^{-1}\left(\eta \circ \phi_{\mathcal{A}}^{-1} * b\right),
\end{array}\right\}
$$

where $b=\phi(a) \in \hat{\mathcal{A}}$. But (acting $\phi$ on $(1.5))$

$$
\left(b * f \circ \phi_{\mathcal{A}}^{-1}\right) M \hat{\Lambda} M^{-1}=M \hat{\Lambda} M^{-1}\left(f \circ \phi_{\mathcal{A}}^{-1} * b\right) .
$$

Thus (4.6) is equivalent to

$$
\hat{p} b=\left\{b *\left[\left(M^{-1} f M\right) \circ \phi_{\mathcal{A}}^{-1}\right]\right\} \hat{p}+
$$




$$
\begin{aligned}
& \frac{1}{c}\left\{b *\left[M^{-1}(\eta+f M h-\epsilon M h) \circ \phi_{\mathcal{A}}^{-1}\right]\right\}- \\
& \frac{1}{c} \hat{\Lambda}\left\{\left[M^{-1}(\eta+f M h-\epsilon M h) \circ \phi_{\mathcal{A}}^{-1}\right] * b\right\} .
\end{aligned}
$$

It proves (4.2) and (4.3). Applying (4.2) and (4.3) to $\hat{\Lambda}$ one obtains (4.5) and

$$
\hat{Z}=\frac{1}{c}\left[\left(M^{-1} \otimes M^{-1}\right) Z M+\hat{R}(\mathbf{1} \otimes h)-h \otimes \mathbf{1}\right] .
$$

Acting $\phi$ on the relation (3.52) and using (4.5), we get

$$
\begin{gathered}
\left(\hat{R}-\mathbf{1}^{\otimes 2}\right)\left[\hat{p} \oplus \hat{p}+\hat{p} \oplus h^{\prime}-\hat{p} \oplus \hat{\Lambda} h^{\prime}+\right. \\
h^{\prime} \oplus \hat{p}-\hat{\Lambda} h^{\prime} \oplus \hat{p}+\hat{\Lambda} h^{\prime} \oplus \hat{\Lambda} h^{\prime}-\hat{\Lambda} h^{\prime} \oplus h^{\prime}-h^{\prime} \oplus \hat{\Lambda} h^{\prime}+h^{\prime} \oplus h^{\prime}- \\
\left.Z^{\prime} \hat{p}-Z^{\prime} h^{\prime}+Z^{\prime} \hat{\Lambda} h^{\prime}+T^{\prime}-(\hat{\Lambda} \oplus \hat{\Lambda}) T^{\prime}\right]=0,
\end{gathered}
$$

where $Z^{\prime}=\frac{1}{c}\left(M^{-1} \otimes M^{-1}\right) Z M, T^{\prime}=\frac{1}{c^{2}}\left(M^{-1} \otimes M^{-1}\right) T, h^{\prime}=\frac{h}{c}$. But

$$
\hat{p} \oplus \hat{\Lambda}=\hat{R}(\hat{\Lambda} \oplus \hat{p})+\hat{Z} \hat{\Lambda}-(\hat{\Lambda} \oplus \hat{\Lambda}) \hat{Z}
$$

so (4.8) is equivalent to

$$
\left(\hat{R}-\mathbf{1}^{\otimes 2}\right)[\hat{p} \oplus \hat{p}-G \hat{p}+U]=0,
$$

where

$$
\begin{gathered}
G=-\mathbf{1} \otimes h^{\prime}+\hat{R}\left(\hat{\Lambda} h^{\prime} \otimes \mathbf{1}\right)-h^{\prime} \otimes \mathbf{1}+\hat{\Lambda} h^{\prime} \otimes \mathbf{1}+Z^{\prime}= \\
\hat{Z}+\left(\hat{R}+\mathbf{1}^{\otimes 2}\right)\left(\hat{\Lambda} h^{\prime} \otimes \mathbf{1}-\mathbf{1} \otimes h^{\prime}\right), \\
U=-\hat{Z} \hat{\Lambda} h^{\prime}+(\hat{\Lambda} \oplus \hat{\Lambda}) \hat{Z} h^{\prime}+\hat{\Lambda} h^{\prime} \oplus \hat{\Lambda} h^{\prime}-\hat{\Lambda} h^{\prime} \oplus h^{\prime}-h^{\prime} \oplus \hat{\Lambda} h^{\prime}+ \\
h^{\prime} \oslash h^{\prime}-Z^{\prime} h^{\prime}+Z^{\prime} \hat{\Lambda} h^{\prime}+T^{\prime}-(\hat{\Lambda} \oplus \hat{\Lambda}) T^{\prime} .
\end{gathered}
$$

But

$$
\begin{gathered}
Z^{\prime} \hat{\Lambda} h^{\prime}-\hat{Z} \hat{\Lambda} h^{\prime}=h^{\prime} \oplus \hat{\Lambda} h^{\prime}-\hat{R}\left(\hat{\Lambda} h^{\prime} \oplus h^{\prime}\right) \\
(\hat{\Lambda} \oplus \hat{\Lambda}) \hat{Z} h^{\prime}=(\hat{\Lambda} \oplus \hat{\Lambda}) Z^{\prime} h^{\prime}+\hat{R}(\hat{\Lambda} \oplus \hat{\Lambda})\left(h^{\prime} \oplus h^{\prime}\right)-(\hat{\Lambda} \oplus \hat{\Lambda}) h^{\prime} \oplus h^{\prime}
\end{gathered}
$$

hence

$$
U=\tilde{T}-(\hat{\Lambda} \oplus \hat{\Lambda}) \tilde{T}+\left(\hat{R}+\mathbf{1}^{\otimes 2}\right)\left(\hat{\Lambda} h^{\prime} \oplus \hat{\Lambda} h^{\prime}-\hat{\Lambda} h^{\prime} \oplus h^{\prime}\right)
$$


where $\tilde{T}=T^{\prime}-Z^{\prime} h^{\prime}+h^{\prime} \oplus h^{\prime}$. Therefore (4.8) is equivalent to

$$
\left(\hat{R}-\mathbf{1}^{\otimes 2}\right)(\hat{p} \oplus \hat{p}-\hat{Z} \hat{p}+\tilde{T}-(\hat{\Lambda} \oplus \hat{\Lambda}) \tilde{T})=0 .
$$

Thus we can choose $\hat{T}$ as $\frac{1}{2}\left(\mathbf{1}^{\otimes 2}-\hat{R}\right) \tilde{T}$ (cf Remark 3.11) and (4.4) follows.

ad 3) We define $\hat{f}, \hat{\eta}, \hat{T}$ by (4.2)-(4.4) and $\hat{\mathcal{B}}$ as the universal algebra generated by $\hat{\mathcal{A}}$ and $\hat{p}_{i}, i \in \mathcal{I}$, satisfying $I_{\hat{\mathcal{B}}}=I_{\hat{\mathcal{A}}}$,

$$
\begin{gathered}
\hat{p} b=(b * \hat{f}) \hat{p}+(b * \hat{\eta})-\hat{\Lambda}(\hat{\eta} * b), \quad b \in \hat{\mathcal{A}}, \\
\left(\hat{R}-\mathbf{1}^{\otimes 2}\right)[\hat{p} \oplus \hat{p}-\hat{Z} \hat{p}+\hat{T}-(\hat{\Lambda} \oplus \hat{\Lambda}) \hat{T}]=0,
\end{gathered}
$$

where $\hat{f}, \hat{\eta}, \hat{T}$ are given by (4.2)-(4.4), $\hat{R}_{i j, k l}=\hat{f}_{i l}\left(\hat{\Lambda}_{j k}\right), \hat{Z}=\hat{\eta}(\hat{\Lambda})$. The computations in 2) show that there exists a unital homomorphism of algebras $\phi: \mathcal{B} \longrightarrow \hat{\mathcal{B}}$ such that $\phi_{\left.\right|_{\mathcal{A}}}=\phi_{\mathcal{A}}$ and $(4.1)$ holds ( $\phi$ transforms the relations in $\mathcal{B}$ into the relations in $\hat{\mathcal{B}}$ ). The same computations show that there exists a unital homomorphism of algebras $\phi^{\prime}: \hat{\mathcal{B}} \longrightarrow \mathcal{B}$ such that $\phi_{\mid \hat{\mathcal{A}}}^{\prime}=\phi_{\mathcal{A}}^{-1}$ and $\phi^{\prime}[M(c \hat{p}+h-\hat{\Lambda} h)]=p$, i.e. $\phi^{\prime}(\hat{p})=\frac{1}{c} M^{-1}[p-M h+\Lambda M h]$. Thus $\phi \phi^{\prime}=\phi^{\prime} \phi=\mathrm{id}$ and $\phi$ is an isomorphism. We set $\hat{\Delta}=(\phi \otimes \phi) \Delta \phi^{-1}$. Hence $(\hat{\mathcal{B}}, \hat{\Delta})$ is a bialgebra with the proper bialgebra structure on $\hat{\mathcal{A}}$ and $\phi$ is an isomorphism of bialgebras. Computations in 2) show $\hat{\Delta} \hat{p}=\hat{p} \oplus I+\hat{\Lambda} \oplus \hat{p}$ and the properties of $(\mathcal{B}, \Delta)$ imply that $(\hat{\mathcal{B}}, \hat{\Delta})$ corresponds to an inhomogeneous quantum group described by $\hat{\mathcal{B}}, \hat{\mathcal{A}}, \hat{\Delta}, \hat{\Lambda}, \hat{p}, \hat{f}, \hat{\eta}, \hat{T}$.

Proposition 4.5 Let $\mathcal{B}, \mathcal{A}, \Delta, \Lambda, p, f, \eta, T$ correspond to an inhomogeneous quantum group where $(\mathcal{A}, \Delta)$ is a Hopf ${ }^{*}$-algebra such that $\bar{\Lambda}=\Lambda$.

1) Let $(\mathcal{B}, \Delta)$ be a Hopf ${ }^{*}$-algebra such that $*_{\left.\right|_{\mathcal{A}}}=*_{\mathcal{A}}$. Then there exist $m \neq$ $0, n_{s} \in \mathbf{C}(s \in \mathcal{I})$ such that

$$
p_{i}^{\prime}=m p_{i}+n_{i}-\Lambda_{i j} n_{j}
$$

satisfy ${p^{\prime}}_{i}^{*}=p^{\prime}{ }_{i}$. In particular, there exist $\hat{\mathcal{B}}, \hat{\mathcal{A}}, \hat{\Delta}, \hat{\Lambda}, \hat{p}, \hat{f}, \hat{\eta}, \hat{T}$ corresponding to an inhomogeneous quantum group and Hopf ${ }^{*}$-algebra isomorphism $\phi$ : $\mathcal{B} \longrightarrow \hat{\mathcal{B}}$ such that $\mathcal{A}=\hat{\mathcal{A}}, \phi_{\left.\right|_{\mathcal{A}}}=\mathrm{id}, \phi\left(p^{\prime}{ }_{i}\right)=\hat{p}_{i}, \hat{p}_{i}^{*}=\hat{p}_{i}$. 
2) There exists Hopf ${ }^{*}$-algebra structure in $\mathcal{B}$ such that $*_{\left.\right|_{\mathcal{A}}}=*_{\mathcal{A}}$ and $p_{i}^{*}=p_{i}$ $(i \in \mathcal{I})$ iff

$$
\begin{aligned}
f_{i j}\left(S\left(a^{*}\right)\right) & =\overline{f_{i j}(a)}, \quad a \in \mathcal{A}, \\
\eta_{i}\left(S\left(a^{*}\right)\right) & =\overline{\eta_{i}(a)}, \quad a \in \mathcal{A}, \\
\tilde{T}-T & \in \operatorname{Mor}(I, \Lambda \oplus \Lambda),
\end{aligned}
$$

where $\tilde{T}_{i j}=\overline{T_{j i}}, i, j \in \mathcal{I}$. Moreover, such $*$ is unique.

3) Proposition 4.4 remains valid if we consider $(\mathcal{A}, \Delta),(\hat{\mathcal{A}}, \hat{\Delta}),(\mathcal{B}, \Delta)$ and $(\hat{\mathcal{B}}, \hat{\Delta})$ as Hopf ${ }^{*}$-algebras, $\phi, \phi_{\mathcal{A}}$ as Hopf ${ }^{*}$-algebra isomorphisms, $p_{i}, \hat{p}_{i}$ as selfadjoints, $M=\bar{M}$ and $c \in \mathbf{R} \backslash\{0\}, h_{i} \in \mathbf{R}(i \in \mathcal{I})$. Moreover, one has $\overline{\hat{\Lambda}}=\hat{\Lambda}$.

Remark 4.6 Statements 1) and 3) remain valid if we replace everywhere Hopf *-algebras by *-bialgebras.

Remark 4.7 If $\Lambda=\bar{\Lambda}, \hat{\Lambda}=\overline{\hat{\Lambda}}$ and $\phi_{\mathcal{A}}: \mathcal{A} \longrightarrow \hat{\mathcal{A}}$ is a ${ }^{*}$-isomorphism such that $\phi_{\mathcal{A}}(\Lambda)=M \hat{\Lambda} M^{-1}$ then we can assume $M=\bar{M}$ (Conjugating one has $\phi_{\mathcal{A}}(\Lambda)=\bar{M} \hat{\Lambda} \bar{M}^{-1}$. Using the condition $b ., \bar{M}=\alpha \cdot M$ for some $\alpha=e^{i \phi}$, $\phi \in \mathbf{R}$. Replacing $M$ by $M^{\prime}=e^{i \phi / 2} M$, one gets $\phi_{\mathcal{A}}(\Lambda)=M^{\prime} \hat{\Lambda} M^{\prime-1}$ and $\left.M^{\prime}=\overline{M^{\prime}}\right)$.

Proof. ad 1) Acting by $*$ on (1.1), we get

$$
\Delta p_{i}^{*}=\Lambda_{i j} \otimes p_{j}^{*}+p_{i}^{*} \otimes I \in \mathcal{A} \otimes \mathcal{B}+\mathcal{B} \otimes \mathcal{A}
$$

Using Proposition 4.3, $p_{i}^{*}=k_{i j} p_{j}+l_{i}$ for some $k_{i j}, l_{i} \in \hat{\mathcal{A}}$. Therefore

$$
(k p+l) \oplus I+\Lambda \oplus(k p+l)=\Delta(k)(p \oplus I+\Lambda \oplus p)+\Delta(l) \text {. }
$$

We get (cf the proof of Proposition 4.4) $k=c I, l=g-\Lambda g$ for some $c, g_{s} \in \mathbf{C}$ $(s \in \mathcal{I})$. Thus $p_{j}^{*}=d p_{j}+g_{j}-\Lambda_{j k} g_{k}$. Using $p_{j}^{* *}=p_{j}$, we may put $d=e^{i \phi}$, $g_{j}=i e^{i \phi / 2} c_{j}, \phi, c_{j} \in \mathbf{R}(j \in \mathcal{I})$. Setting $m=e^{i \phi / 2}, n_{j}=\frac{1}{2} i c_{j}$, one gets that $p_{i}^{\prime}$ given by (4.9) satisfy $p_{i}^{\prime *}=p_{i}^{\prime}$. 
We put $c=\frac{1}{m}, h_{j}=-\frac{1}{m} n_{j}, M=\mathbf{1}, \hat{\mathcal{A}}=\mathcal{A}, \phi_{\mathcal{A}}=$ id. Then $\hat{\mathcal{B}}, \hat{\mathcal{A}}, \hat{\Delta}, \hat{\Lambda}, \hat{p}, \hat{f}, \hat{\eta}, \hat{T}$ and $\phi: \mathcal{B} \longrightarrow \hat{\mathcal{B}}$ given by Proposition 4.4.3 satisfy all the conditions which don't involve $*$. In particular, $\Lambda=\hat{\Lambda}$,

$$
\phi\left(p^{\prime}\right)=\phi(m p+n-\Lambda n)=m(c \hat{p}+h-\Lambda h)+n-\Lambda n=\hat{p} .
$$

We define $*$ in $\hat{\mathcal{B}}$ as $\phi \circ *_{\mathcal{B}} \circ \phi^{-1}$. Then $\phi$ is a Hopf ${ }^{*}$-algebra isomorphism and $\hat{p}_{i}^{*}=\phi\left(p_{i}^{\prime *}\right)=\phi\left(p_{i}^{\prime}\right)=\hat{p}_{i}$.

ad 2) The existence of such structure is equivalent to the fact that the ideal $\mathcal{G}$ in $\tilde{\mathcal{B}}$ (with $*$ given by $\tilde{p}_{i}^{*}=\tilde{p}_{i}, *_{\left.\right|_{\mathcal{A}}}=*_{\mathcal{A}}$ ) generated by (1.6) and (3.46) is selfadjoint (Hopf algebra structure exists due to Proposition 3.12, while $\Delta *=(* \otimes *) \Delta$ and $*^{2}=$ id can be checked on generators $a \in \mathcal{A}, p_{i}(i \in \mathcal{I})$ ). In other words, conjugating (1.6) and (3.46) we should get relations, which follow from (1.6) and (3.46) as relations in algebra without $*$. We use the notation $\bar{f}(x)=\overline{f\left(x^{*}\right)}, f \in \mathcal{A}^{\prime}, x \in \mathcal{A}$. For (1.6) one gets $\left(b=a^{*}\right)$

$$
\begin{gathered}
0=\left[-p_{s} a+\left(a * f_{s t}\right) p_{t}+\phi_{s}(a)\right]^{*}=-b p_{s}+p_{t}\left(b * \overline{f_{s t}}\right)+\phi_{s}\left(b^{*}\right)^{*}= \\
-b p_{s}+\left(b * \overline{f_{s t}} * f_{t r}\right) p_{r}+\phi_{t}\left(b * \overline{f_{s t}}\right)+\phi_{s}\left(b^{*}\right)^{*} .
\end{gathered}
$$

Therefore (see (1.4)) $\overline{f_{s t}} * f_{t r}=\delta_{s r} \epsilon, \overline{f_{s l}}=\overline{f_{s t}} * f_{t r} * f_{r l} \circ S=f_{s l} \circ S, f_{s l}=\overline{f_{s l} \circ S}$, we get (4.10). Moreover,

$$
\phi_{s}\left(b^{*}\right)=-\phi_{t}\left(b * \overline{f_{s t}}\right)^{*} .
$$

Thus

$$
\begin{gathered}
b^{*} * \eta_{s}-\Lambda_{s t}\left(\eta_{t} * b^{*}\right)=-b^{*} * f_{s t} * \overline{\eta_{t}}+\left(\overline{\eta_{m}} * b^{*} * f_{s t}\right) \Lambda_{t m}= \\
-b^{*} * f_{s t} * \overline{\eta_{t}}+\Lambda_{s t}\left(f_{t m} * \overline{\eta_{m}} * b^{*}\right)
\end{gathered}
$$

(we used (1.5)). Thus $\left(b^{*}=a\right) a * \mu_{s}=\Lambda_{s t}\left(\mu_{t} * a\right)$, where $\mu_{s}=\eta_{s}+f_{s t} * \overline{\eta_{t}}$. Inserting $a=v_{k l}, v \in \operatorname{Irr} H$, and denoting $\mu_{s}\left(v_{k m}\right)=F_{s k, m}$, one has $F \in$ $\operatorname{Mor}(v, \Lambda \oplus v)=\{0\}$ (condition c.), $\mu_{s}=0$. Therefore (see (1.4)),

$$
-f_{m s} \circ S * \eta_{s}=f_{m s} \circ S * f_{s t} * \overline{\eta_{t}}=\overline{\eta_{m}} .
$$

Acting on $v_{k l}$ and using (2.5), we obtain

$$
\overline{\eta_{m}}\left(v_{k l}\right)=-f_{m s}\left(v_{k r}^{-1}\right) \eta_{s}\left(v_{r l}\right)=-\eta_{m}\left(\delta_{k l} I\right)+\eta_{m}\left(v_{k r}^{-1}\right) \epsilon\left(v_{r l}\right)=\eta_{m}\left(v_{k l}^{-1}\right),
$$


$\overline{\eta_{m}}=\eta_{m} \circ S$, one gets (4.11).

In virtue of (4.10), (1.4) and $\bar{\Lambda}=\Lambda$

$$
\begin{gathered}
\delta_{i j} \delta_{k l}=\delta_{i j} \epsilon\left(\Lambda_{k l}\right)=f_{i m} * \overline{f_{m j}}\left(\Lambda_{k l}\right)= \\
f_{i m}\left(\Lambda_{k r}\right) \overline{f_{m j}}\left(\Lambda_{r l}\right)=R_{i k, r m} \overline{R_{m r, l j}} .
\end{gathered}
$$

Multiplying by $R_{a b, i k}$, we get

$$
R_{a b, j l}=\overline{R_{b a, l j}}
$$

Moreover,

$$
\eta_{s}\left(\Lambda_{j k}\right)=-\left(f_{s t} * \overline{\eta_{t}}\right)\left(\Lambda_{j k}\right)=-f_{s t}\left(\Lambda_{j m}\right) \overline{\eta_{t}}\left(\Lambda_{m k}\right)=-R_{s j, m t} \overline{\eta_{t}\left(\Lambda_{m k}\right)} .
$$

Multiplying by $\left(R-\mathbf{1}^{\otimes 2}\right)_{a b, s j}$, one obtains

$$
\left(R-\mathbf{1}^{\otimes 2}\right)_{a b, s j} \eta_{s}\left(\Lambda_{j k}\right)=\left(R-\mathbf{1}^{\otimes 2}\right)_{a b, m t} \overline{\eta_{t}\left(\Lambda_{m k}\right)} .
$$

Therefore, conjugating (3.46), we get

$$
\left(R-\mathbf{1}^{\otimes 2}\right)_{l k, j i}\left[p_{j} p_{i}-\eta_{j}\left(\Lambda_{i s}\right) p_{s}+\tilde{T}_{j i}-\Lambda_{j n} \Lambda_{i m} \tilde{T}_{n m}\right]=0 .
$$

Comparing with (3.46), one has $\left(R-\mathbf{1}^{\otimes 2}\right)(\tilde{T}-T) \in \operatorname{Mor}(I, \Lambda \oplus \Lambda)$. Using (3.50) and (4.14), we get (4.12). Conversely, assuming (4.10)-(4.12) and repeating the above reasonings in an opposite order, one gets that $\mathcal{G}$ is selfadjoint.

Since $\mathcal{A}$ and $p_{i}(i \in \mathcal{I})$ generate $\mathcal{B}$, uniqueness of $*$ follows.

ad 3) If $\phi \circ *=* \circ \phi$ and $\bar{M}=M$ then (in 2) of Proposition 4.4)

$$
M \overline{\hat{\Lambda}} M^{-1}=\overline{\phi(\Lambda)}=\phi(\bar{\Lambda})=\phi(\Lambda)=M \hat{\Lambda} M^{-1}
$$

hence $\overline{\hat{\Lambda}}=\hat{\Lambda}$. Moreover,

$$
M(\bar{c} \hat{p}+\bar{h}-\hat{\Lambda} \bar{h})=\overline{\phi(p)}=\phi(\bar{p})=\phi(p)=M(c \hat{p}+h-\hat{\Lambda} h) .
$$

Thus $\bar{h}-h \in \operatorname{Mor}(I, \hat{\Lambda})=\{0\}$ (condition c.), $c, h_{i} \in \mathbf{R}(i \in \mathcal{I})$. In 3) of Proposition 4.4 we prove $\overline{\hat{\Lambda}}=\hat{\Lambda}$ as above and define Hopf *-algebra structure in $\hat{\mathcal{B}}$ by $*_{\hat{\mathcal{B}}}=\phi \circ *_{\mathcal{B}} \circ \phi^{-1}$, which gives the proper $*$ in $\hat{\mathcal{A}}$. Thus $\phi$ is a Hopf ${ }^{*}$-algebra isomorphism by construction. Then

$$
M(c \bar{p}+h-\hat{\Lambda} h)=\overline{\phi(p)}=\phi(\bar{p})=\phi(p)=M(c \hat{p}+h-\hat{\Lambda} h),
$$

$\overline{\hat{p}}=\hat{p}$. 
Proposition 4.8 Let $\mathcal{B}$ satisfy the conditions of Proposition 4.5 .2 with $\tilde{T}=$ T. Then (see (3.54)) $\tau^{k l}\left(S\left(b^{*}\right)\right)=\overline{\tau^{l k}(b)}$.

Proof. Using $\Delta *=(* \otimes *) \Delta, \Delta S=\tau(S \otimes S) \Delta, \epsilon S=\epsilon$, (4.10), (4.11) and $T_{i j}=\overline{T_{j i}}$ one gets

$$
\tau_{i j}\left(S\left(b^{*}\right)\right)=\overline{\eta_{i} * \eta_{j}(b)}-\eta_{i}\left(\Lambda_{j s}\right) \overline{\eta_{s}(b)}+\overline{T_{j i} \epsilon(b)}-\overline{\left(f_{i m} * f_{j n}\right)(b) T_{n m}} .
$$

Multiplying both sides by $\left(R-\mathbf{1}^{\otimes 2}\right)_{k l, i j}$ and using (4.14), (4.15), one gets our assertion.

Corollary 4.9 With assumptions of Proposition 4.8, if (3.55) holds for some $b \in \mathcal{A}$ then (3.55) holds for $S\left(b^{*}\right)$.

Proof. Let $c=S\left(b^{*}\right)$. Applying $S \circ *$ to (3.55) and using Proposition 4.8, one obtains $\left(\Lambda^{-1}\right)_{k i}\left(\Lambda^{-1}\right)_{l j}\left(c * \tau^{j i}\right)=\tau^{l k} * c$, which is equivalent to (3.55) with $b$ replaced by $c$.

\section{Quantum homogeneous spaces}

In this Section we prove that each inhomogeneous quantum group possesses (under some conditions) exactly one analogue of Minkowski space. Throughout the Section we assume that $\operatorname{Poly}(H)=(\mathcal{A}, \Delta)$ is a Hopf *-algebra satisfying the conditions a. - c. and $\operatorname{Poly}(G)=(\mathcal{B}, \Delta)$ is the corresponding Hopf *-algebra as in Theorems 2.1, 3.1 with *-structure as in Proposition 4.5.2.

Remark 5.1 Analogues of Minkowski spaces endowed with the action of inhomogeneous quantum group in absence of inhomogeneous terms in the commutation relations were studied e.g. in [13], 8], [3], for the so called soft deformations (a commutative $\mathcal{A}$ and $\eta=0$ ) in 17 and for $\kappa$-Poincaré group in [y].

Motivated by [12] we say that $(\mathcal{C}, \Psi)$ describes an analogue of Minkowski space associated with $G$ if one has 
1. $\mathcal{C}$ is a unital ${ }^{*}$-algebra generated by $x_{i}, i \in \mathcal{I}$, and $\Psi: \mathcal{C} \longrightarrow \mathcal{B} \otimes \mathcal{C}$ is a unital ${ }^{*}$-homomorphism such that $(\epsilon \otimes$ id $) \Psi=$ id, $($ id $\otimes \Psi) \Psi=(\Delta \otimes$ id $) \Psi$, $x_{i}^{*}=x_{i}$ and

$$
\Psi x_{i}=\Lambda_{i j} \otimes x_{j}+p_{i} \otimes I .
$$

2. if $\Psi W \subset \mathcal{A} \otimes W$ for a linear subspace $W \subset \mathcal{C}$ then $W \subset \mathbf{C} I$.

3. if $\left(\mathcal{C}^{\prime}, \Psi^{\prime}\right)$ also satisfies 1.-2. for some $x_{i}{ }^{\prime} \in \mathcal{C}^{\prime}$ then there exists a unital ${ }^{*}$-homomorphism $\rho: \mathcal{C} \longrightarrow \mathcal{C}^{\prime}$ such that $\rho\left(x_{i}\right)=x_{i}{ }^{\prime}$ and $($ id $\otimes \rho) \Psi=\Psi^{\prime} \rho$ (universality of $(\mathcal{C}, \Psi)$ ).

Let us remark that the conditions $(\epsilon \otimes \mathrm{id}) \Psi=\mathrm{id},(\mathrm{id} \otimes \Psi) \Psi=(\Delta \otimes \mathrm{id}) \Psi$ are superfluous in 1 ..

\section{Proposition 5.2 We assume}

$$
\operatorname{Mor}(I, \Lambda \oplus \Lambda) \cap \operatorname{ker}\left(R+\mathbf{1}^{\otimes 2}\right)=\{0\} .
$$

Let $\mathcal{C}^{\prime}$ be a unital algebra generated by $x_{i}(i \in \mathcal{I})$ and $\Psi^{\prime}: \mathcal{C}^{\prime} \longrightarrow \mathcal{B} \otimes \mathcal{C}^{\prime}$ be a unital homomorphism satisfying (5.1) and the condition 2.. Then

$$
\left(R-\mathbf{1}^{\otimes 2}\right)_{i j, k l}\left(x_{k} x_{l}-\eta_{k}\left(\Lambda_{l m}\right) x_{m}+T_{k l}\right)=0 .
$$

Proof. According to (5.1), $\Psi^{\prime} x=\Lambda \oplus x+p \oplus I$. Thus

$$
\Psi^{\prime}(x \oplus x)=(\Lambda \oplus \Lambda) \oplus(x \oplus x)+(\Lambda \oplus p) \oplus x+(p \oplus \Lambda) \oplus x+(p \oplus p) \oplus I .
$$

Using (1.12), (3.60) and (3.52),

$$
\begin{gathered}
\Psi^{\prime}\left(\left(R-\mathbf{1}^{\otimes 2}\right)(x \oplus x)\right)=(\Lambda \oplus \Lambda) \oplus\left(R-\mathbf{1}^{\otimes 2}\right)(x \oplus x)+ \\
\left(R-\mathbf{1}^{\otimes 2}\right)(Z \Lambda-(\Lambda \oplus \Lambda) Z) \oplus x+\left(R-\mathbf{1}^{\otimes 2}\right)(Z p-T+(\Lambda \oplus \Lambda) T) \oplus I .
\end{gathered}
$$

Setting $w=\left(R-\mathbf{1}^{\otimes 2}\right)(x \oplus x-Z x+T)$, one obtains $\Psi^{\prime} w=(\Lambda \oplus \Lambda) \oplus w$. The condition 2. implies $w_{i j} \in \mathbf{C} I, w=(\Lambda \oplus \Lambda) w$. But $R w=-w$, hence $w \in \operatorname{Mor}(I, \Lambda \oplus \Lambda) \cap \operatorname{ker}\left(R+\mathbf{1}^{\otimes 2}\right)=\{0\}$ and (5.3) follows. 
Proposition 5.3 Assume that

$$
\operatorname{Mor}(I, \Lambda \oplus \Lambda \oplus \Lambda)=\{0\} .
$$

Let $\mathcal{C}$ be the universal unital algebra generated by $x_{i}(i \in \mathcal{I})$ satisfying (5.3). Then there exists a unique unital homomorphism $\Psi: \mathcal{C} \longrightarrow \mathcal{B} \otimes \mathcal{C}$ such that (5.1) holds. Moreover, $\alpha^{i n}\left(x^{\oplus n}\right), i=1,2, \ldots, \operatorname{dim} S_{n}, n=0,1, \ldots, N$, form a basis of

$$
\mathcal{C}^{N}=\operatorname{span}\left\{x_{i_{1}} \cdot \ldots \cdot x_{i_{n}}: i_{1}, \ldots, i_{n} \in \mathcal{I}, n=0,1, \ldots, N\right\},
$$

and the condition 2. holds. In particular,

$$
\operatorname{dim} \mathcal{C}^{N}=\sum_{n=0}^{N} \operatorname{dim} S_{n}
$$

Proof. Doing the same computations as in the proof of Proposition 5.2, we get that the right hand sides of (5.1) satisfy (5.3) in $\mathcal{B} \otimes \mathcal{C}$. Therefore the desired $\Psi$ exists. Uniqueness is trivial. We find the basis of $\mathcal{C}^{N}$ in a similar way as the basis of the left module $\mathcal{B}^{\prime N}$ in Section 3 (Theorem 3.1-Corollary 3.3). The main change is that we now consider the equality $(x \oplus x) \oplus x=x \rrbracket(x \oplus x)$, using $x \oplus x=R(x \oplus x)+k$, where $k=c \cdot x+\left(R-\mathbf{1}^{\otimes 2}\right) T$. One gets $A(k \oplus x)=B(x \oplus k)$. Instead of (3.21)-(3.22) we have

$$
\begin{gathered}
l=-\frac{1}{2} H c, \\
0=-\frac{1}{2} H\left(R-\mathbf{1}^{\otimes 2}\right) T
\end{gathered}
$$

where

$$
l=A\left(\left(R-\mathbf{1}^{\otimes 2}\right) T \otimes \mathbf{1}\right)-B\left(\mathbf{1} \otimes\left(R-\mathbf{1}^{\otimes 2}\right) T\right)=-A_{3}(T \otimes \mathbf{1}-\mathbf{1} \otimes T)=L .
$$

Thus (5.5) is equivalent to (3.21), which is equivalent to (3.1). Moreover, using (3.50), (3.27) and (5.4), one obtains that (5.6) is equivalent to (3.2). Then we find the basis in a similar way as in Section 3.

Now we shall prove the condition 2.. Let $y \in \mathcal{C}, \Psi y \in \mathcal{A} \otimes \mathcal{C}$. If $y \notin \mathbf{C} I$ then for some $N>0$

$$
y=\sum_{i=1}^{\operatorname{dim} S_{N}} c_{i} \alpha^{i N} x^{\oplus N}+y^{\prime}
$$


where $y^{\prime} \in \mathcal{C}^{N-1}$ and not all $c_{i}$ equal 0 . Using (5.1),

$$
\Psi y=\sum_{i=1}^{\operatorname{dim} S_{N}}\left(c_{i} \alpha^{i N} p^{\oplus N}\right) \otimes I+\omega,
$$

where $\omega \in \mathcal{B}^{N-1} \otimes \mathcal{C}$ and also $\Psi y \in \mathcal{B}^{N-1} \otimes \mathcal{C}$. In virtue of Corollary 3.6 $c_{i}=0$. This contradiction shows that $y \in \mathbf{C} I$.

Remark 5.4 Assume (5.9) and (5.4). Then $(\mathcal{C}, \Psi)$ defined in Proposition 5.3 satisfies the conditions 1.-3. without $*$ (one can check $(\epsilon \otimes \mathrm{id}) \Psi=\mathrm{id}$, $(\mathrm{id} \otimes \Psi) \Psi=(\Delta \otimes \mathrm{id}) \Psi,(\mathrm{id} \otimes \rho) \Psi=\Psi^{\prime} \rho$ on generators $)$. The existence of *-structures in $\mathcal{A}, \mathcal{B}$ is not necessary for Proposition 5.9, Proposition 5.3 and Remark 5.4.

Proposition 5.5 Let (5.9) and (5.4) hold and $(\mathcal{C}, \Psi)$ be as in Proposition 5.3. Then there exists a unique ${ }^{*}$-algebra structure in $\mathcal{C}$ such that $\Psi$ is a ${ }^{*}$-homomorphism. It is determined by $x_{i}^{*}=x_{i}$.

Proof. Assume that $\mathcal{C}$ is a ${ }^{*}$-algebra and $\Psi$ is a ${ }^{*}$-homomorphism. Conjugating (5.1) and comparing with (5.1), one gets $\Psi z_{i}=\Lambda_{i j} \otimes z_{j}$ where $z_{i}=x_{i}^{*}-x_{i}$. Using the condition 2. (see Proposition 5.3), $z_{i}=k_{i} I$ with $k_{i} \in$ C. Thus $k=\left(k_{i}\right)_{i=0}^{3} \in \operatorname{Mor}(I, \Lambda)=\{0\}, z_{i}=0, x_{i}^{*}=x_{i}$. Since we must have $I^{*}=I$, it determines $*$ in $\mathcal{C}$ uniquely.

Conversely, setting $x_{i}^{*}=x_{i}$ in free unital algebra generated by $x_{i}$, we get a *-algebra. In virtue of (4.12), (4.14) and (3.50)

$$
\tilde{T}-T \in \operatorname{Mor}(I, \Lambda \oplus \Lambda) \cap \operatorname{ker}\left(R+\mathbf{1}^{\otimes 2}\right)=\{0\} .
$$

Using this, (4.14) and (4.15), one checks that the ideal generated by the left hand sides of (5.3) is selfadjoint. Hence there exists *-algebra structure in $\mathcal{C}$ such that $x_{i}^{*}=x_{i}$. Using (5.1), $\Psi \circ *=* \circ \Psi$ on $x_{i}$, hence in whole $\mathcal{C}$, and $\Psi$ is $\mathrm{a}^{*}$-homomorphism.

Theorem 5.6 Assume (5.2) and (5.4). Then the conditions 1.-3. are satisfied if and only if the pair $(\mathcal{C}, \Psi)$ is *-isomorphic to that defined in Propositions 5.3 and 5.5. 
Proof. According to Propositions 5.3 and 5.5, $(\mathcal{C}, \Psi)$ satisfies the conditions 1.-2.. If $\left(\mathcal{C}^{\prime}, \Psi^{\prime}\right)$ also satisfies 1.-2., then using Proposition 5.2, (5.3) is satisfied in $\mathcal{C}^{\prime}$ and there exists a unital homomorphism $\rho: \mathcal{C} \longrightarrow \mathcal{C}^{\prime}$ such that $\rho\left(x_{i}\right)=x_{i}{ }^{\prime}$. Using $x_{i}^{*}=x_{i}, x_{i}{ }^{\prime}{ }^{*}=x_{i}{ }^{\prime}$, one gets that $\rho \circ *=* \circ \rho$. Using (5.1), one gets $(\mathrm{id} \otimes \rho) \Psi=\Psi^{\prime} \rho$ on $x_{i}$ and hence in whole $\mathcal{C}$. Thus the condition 3 . is satisfied. Uniqueness follows from the universality.

Proposition 5.7 Assume (5.9) and (5.4). Let $\phi: \mathcal{B} \longrightarrow \hat{\mathcal{B}}$ be a Hopf ${ }^{*}$ algebra isomorphism of quantum inhomogeneous groups, $p_{i}^{*}=p_{i}, \hat{p}_{i}^{*}=\hat{p}_{i}$, $\phi_{\left.\right|_{\mathcal{A}}}=\phi_{\mathcal{A}}: \mathcal{A} \longrightarrow \hat{\mathcal{A}}$ be a Hopf ${ }^{*}$-algebra isomorphism such that $\phi_{\mathcal{A}}(\Lambda)=$ $M \hat{\Lambda} M^{-1}, \phi(p)=M(c \hat{p}+h-\hat{\Lambda} h), \bar{M}=M, c, h_{s} \in \mathbf{R}(s \in \mathcal{I})$. Let $(\mathcal{C}, \Psi)$ and $(\hat{\mathcal{C}}, \hat{\Psi})$ be the corresponding objects satisfying 1.-3.. Then there exists a unital $^{*}$-isomorphism $\phi_{\mathcal{C}}: \mathcal{C} \longrightarrow \hat{\mathcal{C}}$ such that $\phi_{\mathcal{C}}(x)=M(c \hat{x}+h)$ and $\phi, \phi_{\mathcal{C}}$ intertwine $\Psi$ with $\hat{\Psi}$.

Proof. We set $\tilde{x}=c^{-1}\left(M^{-1} x-h\right)$ and check that $(\mathcal{C},(\phi \otimes \mathrm{id}) \Psi)$ satisfies the conditions 1.-3. w.r.t. $\tilde{x}, \hat{\Lambda}$ and $\hat{p}$. By virtue of universality, there exists a unital ${ }^{*}$-isomorphism $\phi_{\mathcal{C}}: \mathcal{C} \longrightarrow \hat{\mathcal{C}}$ such that $\phi_{\mathcal{C}}(\tilde{x})=\hat{x},\left(\phi \otimes \phi_{\mathcal{C}}\right) \Psi=\hat{\Psi} \phi_{\mathcal{C}} . \square$. 


\section{Acknowledgements}

The first author is grateful to Prof. W. Arveson and other faculty members for their kind hospitality in UC Berkeley. The authors are thankful to Prof. J. Lukierski and Dr S. Zakrzewski for fruitful discussions.

\section{References}

[1] Brzeziński, T., Remarks on bicovariant differential calculi and exterior Hopf algebras, Lett. Math. Phys. 27 (1993), 287-300.

[2] Celeghini, E., Giachetti, R., Sorace, E. and Tarlini, M., The threedimensional Euclidean quantum group $E_{q}(3)$ and its R-matrix, J. Math. Phys. 32 (1991), 1159-1165.

[3] Chaichian, M. and Demichev, A.P., Quantum Poincaré group, Phys. Lett. B304 (1993), 220-224. Cf also Schirrmacher, A., "Varieties on quantized spacetime" in: "Symmetry methods in physics", A.N. Sissakin et al. (eds.), vol.2, 463-470, Dubna 1994.

[4] Dobrev, V.K., Canonical q-deformations of noncompact Lie (super-) algebras, J.Phys.A: Math. Gen. 26(1993), 1317-1334.

[5] Jimbo, M., A q-analogue of $U(g l(N+1))$, Hecke algebra, and the YangBaxter equation, Lett. Math. Phys. 11(1986), 247-252.

[6] Kondratowicz, P. and Podleś, P., On representation theory of quantum $S L_{q}(2)$ groups at roots of unity, hep-th 9405079, Quantum groups and quantum spaces, Warsaw 1995, Banach Center Publications 40 (1997), 223-248, R. Budzyński et al. (eds.), Inst. of Math., Polish Acad. Sci.

[7] Lukierski, J., Nowicki, A. and Ruegg, H., New quantum Poincaré algebra and $\kappa$-deformed field theory, Phys. Lett. B293 (1992), 344-352; Zakrzewski, S., Quantum Poincaré group related to the $\kappa$-Poincaré algebra, J. Phys. A: Math. Gen. 27 (1994), 2075-2082. Cf also Lukierski,J., Nowicki, A., Ruegg, H. and Tolstoy, V.N., q-deformation of Poincaré algebra Phys. Lett. B264 (1991), 331-338. 
[8] Majid, S., Braided momentum in the q-Poincaré group, J. Math. Phys. 34 (1993), 2045-2058.

[9] Ogievetsky, O., Schmidke, W.B., Wess, J. and Zumino, B., q-Deformed Poincaré algebra, Commun. Math. Phys. 150 (1992), 495-518.

[10] Podleś, P., Complex quantum groups and their real representations, Publ. RIMS, Kyoto University 28 (1992), 709-745.

[11] Podleś, P. and Woronowicz, S.L., "Inhomogeneous quantum groups" in: Proceedings of First Caribbean Spring School of Mathematics and Theoretical Physics, Guadeloupe 1993 (1995), 364-369, World Scientific Singapore-New Jersey-London-Hong Kong.

[12] Podleś, P. and Woronowicz, S.L., On the classification of quantum Poincaré groups, Commun. Math. Phys. 178 (1996), 61-82.

[13] Schlieker, M., Weich, W. and Weixler, R., Inhomogeneous quantum groups, Z. Phys. C. - Particles and Fields 53 (1992), 79-82; Inhomogeneous quantum groups and their universal enveloping algebras, Lett. Math. Phys. 27 (1993), 217-222.

[14] Woronowicz, S.L., Compact matrix pseudogroups, Commun. Math. Phys. 111 (1987), 613-665.

[15] Woronowicz, S.L., Differential calculus on compact matrix pseudogroups (quantum groups), Commun. Math. Phys. 122 (1989), 125-170.

[16] Woronowicz, S.L. and Zakrzewski, S., Quantum deformations of the Lorentz group. The Hopf *-algebra level, Comp. Math. 90 (1994), 211243.

[17] Zakrzewski, S., Geometric quantization of Poisson groups - diagonal and soft deformations, Contemp. Math., vol. 179 (1994), 271-285.

[18] Zakrzewski, S., Poisson structures on the Poincaré group, qalg/9602001, will appear in Commun. Math. Phys.

Note. In Ref. 11 one should assume that $\mathcal{A}_{0}$ has an invertible coinverse. 